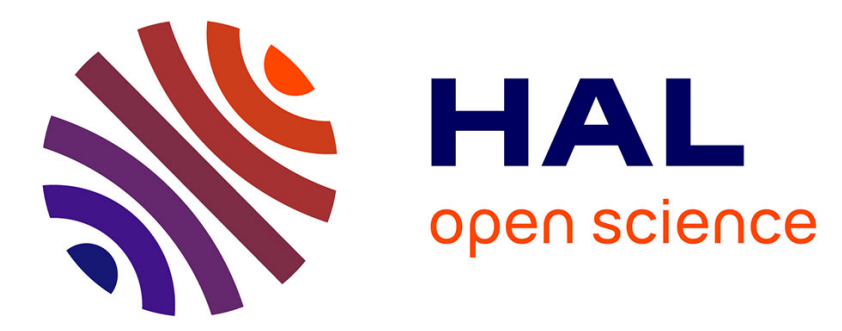

\title{
On the Energy Efficiency of Heterogeneous Cellular Networks With Renewable Energy Sources-A Stochastic Geometry Framework
}

Thanh Tu Lam, Marco Di Renzo

\section{- To cite this version:}

Thanh Tu Lam, Marco Di Renzo. On the Energy Efficiency of Heterogeneous Cellular Networks With Renewable Energy Sources-A Stochastic Geometry Framework. IEEE Transactions on Wireless Communications, 2020, 19 (10), pp.6752-6770. 10.1109/TWC.2020.3005618 . hal-03020407

\section{HAL Id: hal-03020407 https://hal.science/hal-03020407}

Submitted on 24 Nov 2020

HAL is a multi-disciplinary open access archive for the deposit and dissemination of scientific research documents, whether they are published or not. The documents may come from teaching and research institutions in France or abroad, or from public or private research centers.
L'archive ouverte pluridisciplinaire HAL, est destinée au dépôt et à la diffusion de documents scientifiques de niveau recherche, publiés ou non, émanant des établissements d'enseignement et de recherche français ou étrangers, des laboratoires publics ou privés. 


\title{
On the Energy Efficiency of Heterogeneous Cellular Networks with Renewable Energy Sources - A Stochastic Geometry Framework
}

\author{
Thanh Tu Lam, Student Member, IEEE and Marco Di Renzo, Fellow, IEEE
}

\begin{abstract}
In this paper, we introduce an analytical approach for modeling and analyzing the performance of multi-tier cellular networks that are powered by the power grid and by renewable energy sources. The proposed approach relies on modeling the locations of the base stations, either powered by the power grid or by renewable energy sources, by using Poisson point processes. The availability of renewable energy is modeled by using a Poisson point process in the time domain. In particular, the temporal dynamics of the batteries of the base stations powered by renewable energy sources are modeled by using a discrete Markov chain with a number of states that is equal to the finite storage capacity of the batteries. By leveraging recent results available in [1], the coverage probability, the spectral efficiency, and the energy efficiency of the considered network model are formulated in an analytical, closed-form, expression, which depends on the probability that the typical base station is available, i.e., it has sufficient power to serve at least one mobile terminal in its cell. This latter probability is shown to be the solution of the steady state equation of the Markov chain that models the temporal dynamics of the batteries of the base stations. Under the assumption that the batteries of the base stations can be either empty or fully charged, we formulate an optimization problem in order to maximize the energy efficiency as a function of the transmit power and the deployment density of the base stations, and identify sufficient conditions for which the problem admits a unique solution. The accuracy of the proposed approach and the performance trends inferred from it are substantiated with the aid of extensive Monte Carlo simulations.
\end{abstract}

Index Terms-Cellular networks, renewable energy, spectral efficiency, energy efficiency, stochastic geometry.

\section{INTRODUCTION}

Network densification is a key enabler for increasing the spectral efficiency in future wireless networks [2]. Increasing the density of the Base Stations (BSs) in cellular networks increases, however, the network power consumption [1]. Appropriate solutions need, therefore, to be developed in order

Manuscript received May 30, 2019; revised November 16, 2019; and April 23, 2020; accepted June 23, 2020. Date of publication January XY, 2020; date of current version January XY, 2020. This work was supported in part by the European Commission through the H2020-MSCA ETN-5Gwireless project under Grant Agreement 641985. The associate editor coordinating the review of this paper and approving it for publication was K. Huang. (Corresponding author: Marco Di Renzo).

T. T. Lam is with the Xlim Laboratory, Université de Poitiers, 87060 Limoges, France. When this work was done, he was with Université ParisSaclay, CNRS, CentraleSupélec, Laboratoire des Signaux et Systèmes, 91192 Gif-sur-Yvette, France. (e-mail: lam.thanh.tu@xlim.fr).

M. Di Renzo is with Université Paris-Saclay, CNRS, CentraleSupélec, Laboratoire des Signaux et Systèmes, 91192 Gif-sur-Yvette, France. (e-mail: marco.direnzo@12s.centralesupelec.fr).

Digital object identifier XYZ. to leverage the dense and heterogeneous deployment of the BSs in cellular networks without negatively affecting their carbon footprint. A promising solution to reduce the cost of deploying and operating ultra-dense cellular networks consists of employing renewable energy sources, e.g., solar or eolic, to power Small Cell BSs (SBSs), which are overlaid on existing deployments of Macro BSs (MBSs) that are directly connected to the power grid. This approach has the potential of significantly reducing the network power consumption while fulfilling the spectral efficiency and Energy Efficiency (EE) needs [3]. The uncertain availability of renewable energy sources may, however, lead to random fluctuations of the power supplied to the SBSs, which may negatively affect the coverage and spectral efficiency of the overall network. It is, as a result, fundamental to quantify the performance trade-offs in heterogeneous cellular networks powered by renewable energy sources, and to optimize their operation accordingly.

In the present paper, motivated by these considerations, we develop an analytical framework for analyzing and optimizing two-tier cellular networks in which the MBSs are connected to the power grid and the SBSs are powered solely by renewable energy sources. To this end, we capitalize on the mathematical tool of stochastic geometry [4]. Several researchers have recently studied different application scenarios in which renewable energy sources are employed to power cellular BSs. Notable research contributions related to ours include [5]-[12]. Compared with the most closely related research works available in the literature, i.e., [5], [7], [8], [12], in particular, the novelty and contribution of the present paper can be summarized as follows.

Compared with [5], we consider a general Markov chain model to describe the temporal dynamics of the SBSs, which is not restricted to a birth-death process. We study different load and energy harvesting models, and employ a different definition of coverage probability that allows us to formulate and solve optimization problems for some simplified but relevant system models. Compared with [7], we consider a downlink cellular network, and we take into account the network load as a function of the deployment densities of BSs and users. Our system model leads to a different formulation of the Markov chain that describes the energy harvesting dynamics of the batteries of the SBSs. Compared with [8] and [12], we consider a more general Markov chain structure for modeling the temporal dynamics of the batteries of the SBSs, and a power transmission and consumption models that depend on the distribution of the users. 
TABLE I: Main symbols and functions used in the paper $(k \in\{\mathrm{M}, \mathrm{S}\}, d \in\{\mathrm{L} 1, \mathrm{~L} 2\}, o \in\{\mathrm{H}, \mathrm{F}\})$

\begin{tabular}{|c|c|}
\hline Symbol/Function & Definition \\
\hline $\mathbb{E}\{\cdot\}, \operatorname{Pr}\{\cdot\}$ & Expectation operator, probability measure \\
\hline$\lambda_{\mathrm{BS}}, \lambda_{\mathrm{MT}}$ & Reference density of base stations, mobile terminals \\
\hline$\lambda_{k}=\alpha_{k} \lambda_{\mathrm{BS}}, \lambda_{k}^{(\mathrm{A})}$ & Density of tier $k$ ( $\alpha_{k}$ is a constant number), density of available BSs in tier $k$ \\
\hline$\Psi_{k}, \Psi_{k}^{(\mathrm{A})}, \Psi_{\mathrm{MT}}$ & PPP of BSs, available BSs, and MTs in tier $k$ \\
\hline $\mathrm{P}_{\text {tx }, \text { tot }}^{k}, \mathrm{P}_{\text {circ }}^{k}, \mathrm{P}_{\text {idle }}$ & Total transmit, circuits, and idle (only MBSs) power in tier $k$ \\
\hline$P_{\text {unit }}$ & Unit power level of battery storage and power of a packet harvested \\
\hline$r_{n}, g_{n}, g^{(0)}$ & Distance, fading power gain of a generic link and of the intended link \\
\hline$l(\cdot), K_{k}^{(0)}$ & Path-loss, smallest path-loss of the intended link in tier $k$ \\
\hline$\kappa, \beta>0, T_{k}$ & Path-loss constant, slope (exponent), bias factor of tier $k$ \\
\hline $\mathrm{BW}_{\text {tot }}, \mathrm{N}_{0}, \sigma_{\mathrm{N}}^{2}=\mathrm{BW}_{\text {tot }} \mathrm{N}_{0}$ & Total transmission bandwidth, noise power spectral density, noise variance \\
\hline$\gamma_{\mathrm{D}}, \gamma_{\mathrm{A}}, \varepsilon_{d, o}$ & Reliability threshold for decoding, cell association, probability that a SBS is inactive \\
\hline$N_{\text {Load }}^{k}$ & Maximum number of MTs that can be served in tier $k$ (operator-related) \\
\hline$N_{\text {Bat }}^{d}$ & Maximum number of MTs that can be served in tier $k$ (battery-related) \\
\hline$N_{\mathrm{RB}}, \mathrm{AP}_{\mathrm{k}}$ & Number of resource blocks, association probability of tier $k$ \\
\hline $\mathcal{L}, \lambda_{\mathrm{EP}}, p_{u}$ & Battery capacity of SBSs, arrival rate and probability of receiving $u$ power packets \\
\hline$m_{1}^{d}, m^{d}$ & Power levels to make a SBS active, to serve one MT assuming that it is available \\
\hline $\mathbf{R}^{d, o}, \mathbf{v}^{d, o}$ & Matrix of transition probabilities, vector of steady state probabilities \\
\hline$q_{e}^{k}, y_{e}^{k}$ & Probability of having exactly and at least $e$ MTs in a cell of tier $k$ \\
\hline$N_{\mathrm{MT}}, \bar{N}_{\mathrm{MT}}$ & Number of MTs in a cell, number of MTs in a cell given that one is already in it \\
\hline $\mathbf{1}(\cdot),{ }_{2} F_{1}(\cdot, \cdot, \cdot, \cdot), \Gamma(\cdot)$ & Indicator function, Gauss hypergeometric function, gamma function \\
\hline $\max \{x, y\}, \min \{x, y\},\lfloor\cdot\rfloor$ & Maximum, minimum between $x$ and $y$, floor function \\
\hline$\dot{z}(x), \ddot{z}(x)$ & First-order, second-order derivative with respect to $x$ \\
\hline
\end{tabular}

Compared with other papers available in the literature, more importantly, we: (i) propose a tractable approach for performance evaluation that is based on the concept of availability of SBSs, which leads to an implicit formulation of the steady state equation that describes the temporal dynamics of the batteries of the SBSs in which the matrix of transition probabilities depends on the steady state probabilities themselves; (ii) leverage and generalize the new definition of coverage probability recently introduced in [1], [13], [14], which allows us to obtain a tractable closed-form expression of the $\mathrm{EE}$ of two-tier cellular networks in the presence of renewable energy sources; and (iii) formulate and solve EE optimization problems as a function of the transmit power and deployment density of the BSs. We prove, in particular, that the approach introduced in the present paper is general and tractable enough for system-level optimization.

More specifically, we provide the following contributions. (1) We introduce a closed-form analytical framework that quantifies the coverage probability, spectral efficiency, power consumption, and EE in cellular networks where the MBSs are connected to the power grid and the SBSs are powered solely by renewable energy sources. (2) We consider a general system model that accounts for: (i) BSs schedulers that treat the available resources as discrete and continuous; (ii) halfduplex and full-duplex energy harvesting architectures; (iii) power consumption models that depend on the number of Mobile Terminals (MTs) associated with the BSs; (iv) batteries for energy harvesting of finite capacity; and (v) a general Markov chain formulation to model the temporal dynamics of the batteries of the SBSs. (3) We formulate and solve, in simplified but relevant case studies, EE optimization problems, and scrutinize the analytical frameworks to gain insights into the impact of employing renewable energy sources in cellular networks.

The rest of this paper is organized as follows. In Section III. the system model is introduced. In Section III the methodology of analysis and some enabling results for subsequent analysis are presented. In Section IV, the coverage probability, the spectral efficiency, the power consumption, and the EE are formulated in closed-form. In Section V simplified case studies are analyzed in order to gain insight on the impact of renewable energy sources. In Section VI, numerical results are illustrated to validate the proposed approach. Finally, Section VII concludes this paper.

\section{SySTEM MODEL}

In this section, the system model and the modeling assumptions are introduced.

\section{A. Spatial Modeling: Locations of Base Stations and Mobile Terminals}

We consider a two-tier cellular network that consists of MBSs (M) and SBSs (S). The MBSs (denoted also as on-grid BSs) are connected to the power grid while the SBSs (denoted also as off-grid BSs) are powered solely via renewable energy sources. The MBSs and SBSs are distributed according to two independent Poisson Point Processes (PPPs), $\Psi_{\mathrm{M}}$ and $\Psi_{\mathrm{S}}$, whose deployment densities are $\lambda_{\mathrm{M}} \triangleq \alpha_{\mathrm{M}} \lambda_{\mathrm{BS}}$ and $\lambda_{\mathrm{S}} \triangleq \alpha_{\mathrm{S}} \lambda_{\mathrm{BS}}$, where $\alpha_{\mathrm{M}}$ and $\alpha_{\mathrm{S}}$ are positive numbers and 
$\lambda_{\mathrm{BS}}$ is a reference density of BSs. The MTs are distributed according to a homogeneous PPP, $\Psi_{\mathrm{MT}}$, of density $\lambda_{\mathrm{MT}}$, which is independent of $\Psi_{\mathrm{M}}$ and $\Psi_{\mathrm{S}}$. The main symbols and notation used in the paper are available in Table II

\section{B. Temporal Modeling: Energy Harvesting from Renewable Energy Sources}

As for the temporal dynamics of the network, we assume that time is slotted. The duration of the time slot is chosen according to the mobility of the MTs, i.e., the MTs occupy different locations in consecutive time slots. We feel important to note that this assumption is made in the interest of analytical tractability. In general, in fact, the optimization of the time slots in wireless systems depends on a large number of parameters, which include the operating frequency, the type of service to be offered, etc. Without loss of generality, the duration of the time slot is normalized to be unitary. Since the fast fading usually changes on a shorter time scale compared with the locations of the MTs, this implies that the channel gains are independent across the time slots. Under this assumption, the locations of the MBSs and SBSs are considered to be fixed over multiple time slots. The spatial distribution of the MBSs and SBSs is, however, taken into account in the long time horizon [4].

The amount of renewable energy harvested by the SBSs in a time slot is discretized into power packets of fixed power $P_{\text {unit }}$. The number of power packets harvested across the time slots are independent and identically distributed Poisson random variables with mean $\lambda_{\mathrm{EP}}$. The power packets are stored in batteries of finite capacity with $\mathcal{L}$ power levels. Each battery level is equal to $P_{\text {unit }}$, so that the maximum amount of power that can be stored is $(\mathcal{L}-1) P_{\text {unit. }}$. The probability that $z$ power packets are harvested in one time slot is $p_{z} \triangleq\left(\lambda_{\mathrm{EP}}\right)^{z} \exp \left(-\lambda_{\mathrm{EP}}\right) / z$ ! for $z=0,1, \ldots, \mathcal{L}-2$, and $p_{\mathcal{L}-1} \triangleq 1-\sum_{z=0}^{\mathcal{L}-2} p_{z}$ is the probability of harvesting at least $\mathcal{L}-1$ power packets, i.e., the maximum storage capacity. By appropriately choosing $\lambda_{\mathrm{EP}}$, different time scales for the mobility of the MTs and the rate at which the renewable energy is harvested can be modeled, e.g., (i) if $\lambda_{\mathrm{EP}} \gg 1$, the time scale of energy harvesting is shorter that the time scale that corresponds to the mobility of the MTs; (ii) if $\lambda_{\mathrm{EP}} \ll 1$, the opposite holds true. As detailed in the sequel, the temporal dynamics of the batteries of the SBSs are modeled by using a discrete Markov chain with $\mathcal{L}$ states. Based on the considered assumptions, for ease of writing, the time index can be omitted in our notation. It is worth mentioning that the Poisson distribution for modeling the packets of harvested power is not only convenient from the analytical point of view, and, hence, widely employed in the literature, but it is accurate enough for representing empirical data as well. For example, the authors of [15] have shown that a Poisson distribution with an arbitrary transformation (e.g., a translation) well matches empirical measurements of solar-powered energy harvesting.

\section{Availability of Small Cell Base Stations}

For ease of description, we anticipate an important concept: The availability of the SBSs. Since the number of power packets that are harvested by the SBSs in a time slot is a Poisson random variable, some SBSs may not have sufficient power to serve the MTs located in their cells. The SBSs that have sufficient power to serve at least one $M T$ are referred to as available. Otherwise, they are referred to as unavailable. The point process of available SBSs is denoted by $\Psi_{\mathrm{S}}^{(\mathrm{A})} \subseteq \Psi_{\mathrm{S}}$. Since the MBSs are connected to the power grid, we have $\Psi_{\mathrm{M}}^{(\mathrm{A})}=\Psi_{\mathrm{M}}$, i.e., the MBSs are always available. The MTs located in the cells of unavailable SBSs are offloaded either to an available SBS or to an MBS, according to the cell association described next. It is worth noting that the set of available SBSs is, in general, different in every time slot.

\section{Channel Modeling}

In each link, small-scale and large-scale channel impairments are accounted for. All links are independently and identically distributed. The power gain of link $n$ (in both tiers) is denoted by $g_{n}$, and it is an exponential random variable with unit mean. The path-loss of link $n$ in tier $k \in\{\mathrm{M}, \mathrm{S}\}$ and length $r_{n}$ is $l_{k}\left(r_{n}\right) \triangleq \kappa_{k} r_{n}^{\beta_{k}}$, where $\beta_{k}$ and $\kappa_{k}$ are the path-loss exponent and constant of the $k$ th tier. For simplicity, we assume $\beta_{k}=\beta$ and $\kappa_{k}=\kappa$ for $k \in\{\mathbf{M}, \mathbf{S}\}$.

\section{E. Cell Association}

The typical MT, $\mathrm{MT}^{(0)}$, is served by the BS that provides the highest biased average received power to it. Let $T_{k}$ and $P_{\mathrm{tx}, \text { tot }}^{k}$ be the bias factor and the total transmit power of tier $k$, and $K_{k}^{(0)} \triangleq \min _{n \in \Psi_{k}^{(\mathrm{A})}}\left\{l_{k}\left(r_{n}\right)\right\}$ be the smallest path loss of tier $k$, where $k \in\{\mathrm{M}, \mathrm{S}\}$. The average (over the smallscale fading) received power at $\mathrm{MT}^{(0)}$ can be formulated as follows:

$$
P_{\mathrm{rx}}^{(0)} \triangleq \frac{P_{\mathrm{tx}, \mathrm{tot}}^{k}}{K_{k}^{(0)}} \quad \text { if } \quad \frac{T_{\widetilde{k}} K_{\widetilde{k}}^{(0)}}{P_{\mathrm{tx}, \mathrm{tot}}^{\widetilde{k}}} \geq \frac{T_{k} K_{k}^{(0)}}{P_{\mathrm{tx}, \mathrm{tot}}^{k}}
$$

where $\widetilde{k}$ is defined as $\widetilde{k}=\mathrm{S}$ if $k=\mathrm{M}$ and $\widetilde{k}=\mathrm{M}$ if $k=\mathrm{S}$, respectively.

Since the locations of the MTs and the available SBSs may be different in different time slots, the typical MT may, based on (1), be served by a different BS in different time slots.

\section{F. Load Model}

Let $N_{\mathrm{MT}}$ be the number of MTs in a generic cell. Two load models are analyzed.

1) Load Model 1 (L1): Power and bandwidth are viewed as discrete resources by the scheduler: The total transmit power and total transmission bandwidth of the MBSs and SBSs are split into $N_{\mathrm{RB}} \in \mathbb{N}$ discrete units, which are referred to as Resource Blocks (RBs). Within a cell, each MBS and SBS can serve only one MT in each RB. Therefore, intracell interference is avoided. All MBSs and SBSs are allowed to transmit in any RB. Therefore, inter-cell interference is present on a per-RB basis. The transmit power and transmission bandwidth of each $\mathrm{RB}$ are $P_{\mathrm{tx}}^{k, \mathrm{~L} 1} \triangleq P_{\mathrm{tx}, \mathrm{tot}}^{k} / N_{\mathrm{RB}}$ and $\mathrm{BW}_{k, \mathrm{~L} 1} \triangleq \mathrm{BW}_{\mathrm{L} 1}=\mathrm{BW}_{\text {tot }} / N_{\mathrm{RB}}$, respectively, where $\mathrm{BW}_{\text {tot }}$ is the total transmission bandwidth. Under this model, 
the maximum number of MTs that can be served by each MBS and SBS is $N_{\text {max }}^{\mathrm{M}, \mathrm{L} 1} \triangleq \min \left\{N_{\mathrm{RB}}, N_{\mathrm{Load}}^{\mathrm{M}}\right\}$ and $N_{\max }^{\mathrm{S}, \mathrm{L} 1} \triangleq$ $\min \left\{N_{\mathrm{RB}}, N_{\text {Load }}^{\mathrm{S}}, N_{\text {Bat }}^{\mathrm{L} 1}\right\}$, respectively, where $N_{\text {Load }}^{k} \in \mathbb{N}$ for $k \in\{\mathrm{M}, \mathrm{S}\}$ is a free parameter for resource allocation, e.g., to control the inter-cell interference in the RBs, and $N_{\text {Bat }}^{\mathrm{L} 1} \in \mathbb{N}$ denotes the maximum number of MTs that can be served only due to the random arrival of the power packets and the limited storage capacity of the battery of the SBSs. Further details on $N_{\mathrm{Bat}}^{\mathrm{L} 1}$ are given in the sequel. If $N_{\mathrm{MT}}>N_{\max }^{\mathrm{M}, \mathrm{L} 1}$ or $N_{\mathrm{MT}}>N_{\max }^{\mathrm{S}, \mathrm{L} 1}$, some MTs cannot be served in a given time slot. In this case, only $N_{\max }^{\mathrm{M}, \mathrm{L} 1}$ or $N_{\max }^{\mathrm{S}, \mathrm{L} 1}$ MTs are served, and they are chosen uniformly at random among the $N_{\mathrm{MT}}$ MTs that are available. If $N_{\mathrm{MT}}<N_{\max }^{\mathrm{M}, \mathrm{L} 1}$ or $N_{\mathrm{MT}}<N_{\max }^{\mathrm{S} \text {,L1 }}$, some MBSs and SBSs do not transmit in some RBs, thus generating less inter-cell interference and consuming less power.

2) Load Model 2 (L2): Power and bandwidth are viewed as continuous resources by the scheduler: The total transmit power and total transmission bandwidth of the MBSs and SBSs are viewed as continuous resources, and are evenly allocated among the maximum number of MTs that can be served by each MBS and SBS. Similar to L1, however, no intra-cell interference is present. By using a notation similar to L1, the maximum number of MTs that can be served by each MBS and SBS is $N_{\max }^{\mathrm{M}, \mathrm{L} 2} \triangleq \min \left\{N_{\mathrm{MT}}, N_{\text {Load }}^{\mathrm{M}}\right\}$ and $N_{\max }^{\mathrm{S}, \mathrm{L} 2} \triangleq \min \left\{N_{\mathrm{MT}}, N_{\mathrm{Load}}^{\mathrm{S}}, N_{\mathrm{Bat}}^{\mathrm{L} 2}\right\}$, respectively. Therefore, the transmission bandwidth allocated by each MBS and SBS to each MT is $\mathrm{BW}_{k, \mathrm{~L} 2} \triangleq \mathrm{BW}_{\text {tot }} / N_{\max }^{k, \mathrm{~L} 2}$, and the total transmit power of each MBS and SBS is evenly spread across the entire transmission bandwidth $\mathrm{BW}_{\text {tot }}$, i.e., the power spectral density is $P_{\mathrm{tx}, \mathrm{tot}}^{k} / \mathrm{BW}_{\text {tot }}$ for $k \in\{\mathrm{M}, \mathrm{S}\}$. L1 and L2 are similar, but two main differences hold. In L2: (i) the transmit power and transmission bandwidth that are allocated to each MT are random variables (since $N_{\mathrm{MT}}$ is a random variable); and (ii) each MBS and SBS consumes the total transmit power and uses the entire transmission bandwidth if $N_{\mathrm{MT}} \geq 1$ (at each transmission instance).

In simple terms, therefore, two models for the load and two models for the corresponding transmit power of the MBSs and SBSs are studied. As for load model L1, the total available bandwidth and the total transmit power are split into RBs of fixed size. Each MT is served on an independent RB in order to avoid intra-cell interference. The number of MTs that can be served by an MBS depends, therefore, on the total number of RBs. The number of MTs that can be served by an SBS depends, on the other hand, on the total number of RBs and on the energy harvesting process (i.e., the random arrival of the power packets and the limited storage capacity of the battery of the SBSs). This implies that some MTs may not be served under load model L1. As for the load model L2, we consider that the total available bandwidth is equally split among all the MTs that lie in a cell. Similarly, the total transmit power is spread across the total available bandwidth. This ensures that all the MTs that lie in a cell are served, but without guaranteeing any minimum constraint on the bandwidth and transmit power that are allocated to them.

\section{G. Power Transmission and Consumption Modeling}

The MBSs and SBSs operate in two modes. The MBSs can be either in transmit or idle mode if at least one MT or no MT is associated to them, respectively. In the first case, the power consumed is the summation of the transmit power $P_{\mathrm{tx}}^{\mathrm{M}, d}$ for $d \in\{\mathrm{L} 1, \mathrm{~L} 2\}$ which is defined in the previous section, and the circuits power consumption $P_{\text {cir }}^{\mathrm{M}}$ [1]. In idle mode, the power consumption is $0 \leq P_{\text {idle }}^{\mathrm{M}} \leq P_{\text {cir }}^{\mathrm{M}}$. The SBSs can be either in transmit or energy harvesting mode. They operate in transmit mode if at least one MT is associated to them and they have sufficient power in their batteries to serve at least one MT (i.e., they are available). They operate in energy harvesting mode if either no MT is associated to them or they are unavailable. In the first case, similar to the MBSs, the power consumed is the summation of the transmit power $P_{\mathrm{tx}}^{\mathrm{S}, d}$ for $d \in\{\mathrm{L} 1, \mathrm{~L} 2\}$, and the circuits power consumption $P_{\text {cir }}^{\mathrm{S}}$. The operation in the energy harvesting mode is detailed in the next section. For simplicity, we adopt the notation $P_{\text {idle }}=P_{\text {idle }}^{\mathrm{M}}$.

In general terms, therefore, the power consumption of the MBSs and SBSs is obtained as the sum of the transmit power, the circuits power, and the idle power. The main difference between the MBSs and SBSs is that the idle power is assumed to be equal to zero for the SBSs. The power consumption of the SBSs is equal to zero during the harvesting process.

\section{H. Energy Harvesting Modeling}

1) Definition of $N_{\text {Bat }}^{d}$ for $d \in\{L 1, L 2\}$ : As anticipated, the amount of renewable energy harvested by the SBSs is discretized into packets of fixed power $P_{\text {unit }}$, and each power level of the batteries is, for simplicity, equal to $P_{\text {unit }}$. Based on the power consumption model introduced in the previous section, we can formally define $N_{\text {Bat }}^{d} \in \mathbb{N}$, i.e., the maximum number of MTs that can be served by the SBSs by taking into account only the random arrival of the power packets and the limited storage capacity of the SBSs. Let $\widetilde{P}_{\text {tx } \text {,tot }}^{\mathrm{S}} \triangleq\left\lfloor P_{\mathrm{tx}, \text { tot }}^{\mathrm{S}} / P_{\text {unit }}\right\rfloor \in \mathbb{N}$ and $\widetilde{P}_{\text {cir }}^{\mathrm{S}} \triangleq\left\lfloor P_{\text {cir }}^{\mathrm{S}} / P_{\text {unit }}\right\rfloor \in \mathbb{N}$ be the number of power levels that correspond to $P_{\mathrm{tx}, \text { tot }}^{\mathrm{S}}$ and $P_{\text {cir }}^{\mathrm{S}}$, respectively. Then, $N_{\text {Bat }}^{d} \triangleq\left\lfloor\left(\mathcal{L}-1-m_{1}^{d}\right) / m^{d}\right\rfloor+1$, where $m_{1}^{d} \in \mathbb{N}$ and $m^{d} \in \mathbb{N}$ are the minimum power level that is necessary to make an SBS available and the minimum power level that is necessary to serve one MT conditioned on the availability of the SBS, respectively. We have: $m_{1}^{\mathrm{L} 1}=\widetilde{P}_{\mathrm{cir}}^{\mathrm{S}}+\widetilde{P}_{\mathrm{tx}, \text { tot }}^{\mathrm{S}} / N_{\mathrm{RB}}, m_{1}^{\mathrm{L} 2}=\widetilde{P}_{\mathrm{cir}}^{\mathrm{S}}+\widetilde{P}_{\mathrm{tx}, \mathrm{tot}}^{\mathrm{S}}$, $m^{\mathrm{L} 1}=\widetilde{P}_{\text {cir }}^{\mathrm{S}}+\widetilde{P}_{\mathrm{tx} \text {,tot }}^{\mathrm{S}} / N_{\mathrm{RB}}$, and $m^{\mathrm{L} 2}=\widetilde{P}_{\text {cir }}^{\mathrm{S}}$. The definitions of $m_{1}^{d}$ and $m^{d}$ account for the different assumptions under L1 and L2. Under L2, the total transmit power is taken into account only in $m_{1}^{\mathrm{L} 2}$, since it is entirely used even if a single MT is served. This is the reason why $m^{\mathrm{L} 2}$ depends only on the circuits power consumption.

2) Half-Duplex and Full-Duplex Energy Harvesting: In energy harvesting mode, the SBSs can operate in either halfduplex $(\mathrm{H})$ or full-duplex $(\mathrm{F})$. In the first case, the SBSs need a single battery that, in every time slot, is in either transmission mode or energy harvesting mode. In the second case, on the other hand, the SBSs can transmit and harvest energy during every time slot. In full-duplex mode, the SBSs are equipped 
with two batteries. One of them is used for transmission and the other one is used for energy harvesting. At the end of the time slot, the latter battery transfers the harvested power to the former battery instantly. Therefore, the power harvested in a given time slot can be used for transmission only in subsequent time slots.

\section{Methodology of AnAlysis And Preliminary RESULTS}

In this section, the main methodology of analysis and some enabling results are presented.

\section{A. Methodology of Analysis}

The MTs and the power packets harvested by the SBSs are distributed according to two time-homogeneous (stationary in time) and spatial-homogeneous (stationary in space) independent PPPs. For a given network realization of MBSs and SBSs, therefore, the availability of an arbitrary SBS in a given time slot depends on: (i) the power level of its battery in the previous time slot and the amount of harvested power in the previous time slot, if it was unavailable in the previous time slot, and (ii) the power level of its battery in the previous time slot, the number of MTs that it served in the previous time slot, and the amount of harvested power in the previous time slot (only for full-duplex energy harvesting), if it was available in the previous time slot.

The availability of an arbitrary SBS in a given time slot depends, in addition, on the availability of the other SBSs in the previous time slot. Assume, for example, that only one SBS is available in a given time slot. Based on the considered cell association, the SBS has to serve all the MTs in the network. This operation increases the likelihood of depleting the battery of the SBS, and, thus, decreases the likelihood of the SBS to be available in the next time slot. The spatial-temporal coupling that originates from these interactions is difficult to model without resorting to some approximations [16]. Some papers where the spatio-temporal coupling is analyzed can be found in [19], [20], [21]. In our system model, however, the stationarity in time and space of the MTs and the random arrivals of the power packets weaken, in part, the spatiotemporal coupling among the SBSs. For analytical tractability, therefore, the spatio-temporal correlations among the SBSs are not taken into account in our analytical framework, as formally stated below.

Approximation 1: In every time slot, the point process of the available SBSs, $\Psi_{\mathrm{S}}^{(\mathrm{A})}$, is approximated by an independently thinned version of $\Psi_{\mathrm{S}}$, whose retaining probability is the probability that an SBS is available, i.e., the probability that the SBS has sufficient power in its battery to serve at least one MT. By denoting with $\varepsilon_{d, o}$ the probability that an SBS is unavailable, the point process $\Psi_{\mathrm{S}}^{(\mathrm{A})}$ is approximated with a PPP of density $\lambda_{\mathrm{S}}^{(\mathrm{A})} \triangleq\left(1-\varepsilon_{d, o}\right) \lambda_{\mathrm{S}}$.

Remark 1: It is worth mentioning that thinning has been extensively employed when modeling cellular networks with the aid of stochastic geometry. An example is the analysis of uplink cellular networks, where thinning is used to take into account the non-uniform spatial distribution of the MTs that are scheduled for transmission on a given physical resource [22]. Our application of thinning is, however, different and peculiar to the specific problem that we intend to solve. It will be apparent in the sequel, in fact, that $\varepsilon_{d, o}$ jointly depends on the spatial and temporal characteristics of the cellular network. The computation of $\varepsilon_{d, o}$ is, in particular, challenging and, in general, no explicit closed-form expression exists for it (see, e.g., (9)).

Approximation 2: To compute $\varepsilon_{d, o}$, our approach relies on assuming that the discrete Markov chain that describes the temporal dynamics of the batteries of the SBSs operates at the steady state, i.e., at the equilibrium. The discrete Markov chain, therefore, can be modeled via its equilibrium distribution, i.e., the probabilities that the power level of the battery is $\left\{0, P_{\text {unit }}, 2 P_{\text {unit }}, \ldots, \quad(\mathcal{L}-1) P_{\text {unit }}\right\}$ in the long time horizon. Under this assumption, the probability that an SBS is available, i.e., $1-\varepsilon_{d, o}$, is computed at the steady state (equilibrium) of the discrete Markov chain. Our mathematical approach, therefore, ignores the transient behavior of the discrete Markov chain, but it is shown to be analytically tractable and sufficiently accurate. It is worth noting, however, that this transient behavior is taken into account in our Monte Carlo simulations. We anticipate that the proposed approach leads to a non-trivial formulation of the steady state probabilities of the discrete Markov chain, since the transition probabilities implicitly depend on the steady state probabilities through the probability $\varepsilon_{d, o}$. To the best of our knowledge, the proposed analytical formulation has never been employed and analyzed in prior related works.

Remark 2: For analytical tractability, the batteries of the SBSs are assumed to be statistically equivalent. Thus, a single Markov chain is employed. This is, in part, justified by the spatial and temporal independence of the packets of harvested energy, the locations of the MTs, and the fast fading. We feel important to emphasize, however, that this modeling assumption results in approximated analytical frameworks, since the spatial coupling among the locations of the MBSs and SBSs has a stronger impact as compared with, e.g., fast fading. In general, therefore, the batteries of the SBSs exhibit a different statistical behavior across the entire network. Recent research results tackling this issue can be found in [16]-[18].

Remark 3: In the event that no SBS is available in a given network realization, the MTs are served by the MBSs. The MBSs are, in fact, always available as they are connected to the power grid. In the worst case, thus, the system model boils down to a single-tier cellular network.

\section{B. Preliminary Results}

In this section, based on the proposed analytical methodology, we summarize some enabling results that are useful for formulating the analytical frameworks in the next section.

Lemma 1: Consider $\widetilde{k} \neq k$ for $k \in\{\mathrm{M}, \mathrm{S}\}, d \in\{\mathrm{L} 1, \mathrm{~L} 2\}$, and $o \in\{\mathrm{H}, \mathrm{F}\}$. The probability, $\operatorname{AP}_{k} \triangleq \operatorname{AP}_{k}\left(\varepsilon_{d, o}\right)$, that the typical MT is associated with the $k$ th tier is as follows:

$$
\mathrm{AP}_{k} \triangleq \operatorname{Pr}\left(\frac{T_{\widetilde{k}}}{P_{\mathrm{tx}, \mathrm{tot}}^{\widetilde{k}}} K_{\widetilde{k}}^{(0)} \geq \frac{T_{k}}{P_{\mathrm{tx}, \mathrm{tot}}^{k}} K_{k}^{(0)}\right)
$$


TABLE II: Auxiliary functions used throughout this paper $(k \in\{\mathrm{M}, \mathrm{S}\}, d \in\{\mathrm{L} 1, \mathrm{~L} 2\}, o \in\{\mathrm{H}, \mathrm{F}\})$

\begin{tabular}{|l|}
\hline \multicolumn{1}{|c|}{ Function Definition } \\
\hline \hline$\eta=\kappa \gamma_{A} \sigma_{\mathrm{N}}^{2}, \quad N_{\mathrm{LB}}=\min \left\{N_{\mathrm{Load}}^{\mathrm{S}}, N_{\mathrm{Bat}}^{\mathrm{L} 1}\right\}, \quad w_{k}=\left(P_{\mathrm{tx}, \mathrm{tot}}^{k} / \eta\right)^{2 / \beta}$ \\
$c(x)={ }_{2} F_{1}(1,-2 / \beta, 1-2 / \beta,-x)-1$ \\
$a_{1}(k, n)=(3.5)^{3.5} \Gamma(4.5+n)\left(\lambda_{\mathrm{MT}} \mathcal{A}_{k}\right)^{n+1} /\left(\Gamma(3.5) \Gamma(2+n)\left(\lambda_{\mathrm{MT}} \mathcal{A}_{k}+3.5\right)^{n+4.5}\right)$ \\
$a_{2}(a, b, c, k)={ }_{2} F_{1}\left(a, b, c, \lambda_{\mathrm{MT}} \mathcal{A}_{k} /\left(\lambda_{\mathrm{MT}} \mathcal{A}_{k}+3.5\right)\right)$ \\
$f_{1}(d, o, i)=\sum_{s=m_{1}^{d}+(i-1) m^{d}}^{m_{1}^{d}+i m^{d}} v_{-o}^{d, o}, \quad f_{2}(d, o, n)=\sum_{s=m_{1}^{d}+(n-1) m^{d}} v_{s}^{d, o}$ \\
$z_{\text {inc }}^{1}(k, n)=a_{1}(k, n) a_{2}(1,4.5+n, 2+n, k)+a_{1}(k, n) \frac{(n+4.5)\left(\lambda_{\mathrm{MT}} \mathcal{A}_{k}\right)}{(n+2)\left(\lambda_{\mathrm{MT}} \mathcal{A}_{k}+3.5\right)} a_{2}(2,5.5+n, 3+n, k)$ \\
$z_{\text {inc }}^{2}(k, n)=a_{1}(k, n) a_{2}(2,4.5+n, 2+n, k)$ \\
$z_{\mathrm{sel}}(k, n)=1-\left(a_{1}(k, n) /\left(\lambda_{\mathrm{MT}} \mathcal{A}_{k}\right)\right)\left((n+1) a_{2}(1,4.5+n, 1+n, k)-n a_{2}(1,4.5+n, 2+n, k)\right)$ \\
\hline
\end{tabular}

$$
=\left(1+\frac{\lambda_{\widetilde{k}}^{(\mathrm{A})}}{\lambda_{k}^{(\mathrm{A})}}\left(\frac{T_{k}}{T_{\widetilde{k}}} \frac{P_{\mathrm{tx}, \mathrm{tot}}^{\widetilde{k}}}{P_{\mathrm{tx}, \mathrm{tot}}^{k}}\right)^{2 / \beta}\right)^{-1}
$$

Proof: It follows from (1) using analytical steps similar to [23]. The difference is that the point process of the available SBSs is approximated by a PPP of density $\lambda_{\mathrm{S}}^{(\mathrm{A})}$.

Lemma 2: Consider $\mathcal{A}_{k} \triangleq \mathcal{A}_{k}\left(\varepsilon_{d, o}\right)=\mathrm{AP}_{k} / \lambda_{k}^{(\mathrm{A})}$ for $k \in$ $\{\mathrm{M}, \mathrm{S}\}, d \in\{\mathrm{L} 1, \mathrm{~L} 2\}$, and $o \in\{\mathrm{H}, \mathrm{F}\}$. The probability, $q_{e}^{k}$, that exactly e MTs, and the probability, $y_{e}^{k}$, that at least $e$ MTs are associated with a BS of the $k$ th tier can be formulated, respectively, as follows:

$$
\begin{gathered}
q_{e}^{k} \triangleq \operatorname{Pr}(\text { Number of MTs }=e, k) \\
=\frac{(3.5)^{3.5} \Gamma(e+3.5)\left(\lambda_{\mathrm{MT}} \mathcal{A}_{k}\right)^{e}}{\Gamma(3.5) e !\left(\lambda_{\mathrm{MT}} \mathcal{A}_{k}+3.5\right)^{e+3.5}} \\
y_{e}^{k} \triangleq \operatorname{Pr}(\text { Number of MTs } \geq e, k) \\
=1-\sum_{i=0}^{e-1} \frac{(3.5)^{3.5} \Gamma(i+3.5)\left(\lambda_{\mathrm{MT}} \mathcal{A}_{k}\right)^{i}}{\Gamma(3.5) i !\left(\lambda_{\mathrm{MT}} \mathcal{A}_{k}+3.5\right)^{i+3.5}}
\end{gathered}
$$

Proof: The proof of $q_{e}^{k}$ follows by using the approximation for the area of a Poisson-Voronoi cell in [24], and the approximation for the area, $\mathcal{A}_{k}$, of a multi-tier Poisson cellular network, i.e., $\mathcal{A}_{k}=\mathrm{AP}_{k} / \lambda_{k}^{(\mathrm{A})}$ in [25]. Also, the point process of the available SBSs is approximated with a PPP of density $\lambda_{\mathrm{S}}^{(\mathrm{A})}$. The proof of $y_{e}^{k}$ follows from its definition, i.e., $y_{e}^{k}=1-\sum_{i=0}^{e-1} q_{i}^{k}$.

Lemma 3: Consider $\mathcal{A}_{k}=\mathcal{A}_{k}\left(\varepsilon_{d, o}\right)=\mathrm{AP}_{k} / \lambda_{k}^{(\mathrm{A})}$ for $k \in$ $\{\mathrm{M}, \mathrm{S}\}, d \in\{\mathrm{L} 1, \mathrm{~L} 2\}$, and $o \in\{\mathrm{H}, \mathrm{F}\}$. The probability, $\bar{q}_{e}^{k}$, that exactly e MTs are associated with a BS of the $k$ th tier conditioned on one MT being associated with the BS already can be formulated as follows:

$$
\begin{aligned}
\bar{q}_{e}^{k} & \triangleq \operatorname{Pr}\{\text { Number of MTs }=e \mid \mathrm{MT}=1, k\} \\
& =\frac{3.5^{3.5} \Gamma(e+4.5)}{\Gamma(3.5) e !} \frac{\left(\lambda_{\mathrm{MT}} \mathcal{A}_{k}\right)^{e}}{\left(3.5+\lambda_{\mathrm{MT}} \mathcal{A}_{k}\right)^{e+4.5}}
\end{aligned}
$$

Proof: It is similar to Lemma 2, with the exception that the distribution of the area of a Poisson-Voronoi cell conditioned on the presence of one MT is different [24].

Lemma 4: Consider $\mathcal{A}_{k}=\mathcal{A}_{k}\left(\varepsilon_{d, o}\right)=\mathrm{AP}_{k} / \lambda_{k}^{(\mathrm{A})}$ for $k \in$ $\{\mathrm{M}, \mathrm{S}\}, d \in\{\mathrm{L} 1, \mathrm{~L} 2\}$, and $o \in\{\mathrm{H}, \mathrm{F}\}$. Let $a_{1}(\cdot)$ and $a_{2}(\cdot)$ be the functions defined in Table III The average number of
MTs to be served by a BS of the $k$ th tier can be formulated as follows:

$$
\begin{gathered}
\bar{N}_{\mathrm{MT}}^{k, d} \triangleq \mathbb{E}\{\text { Number of MTs }\}=\lambda_{\mathrm{MT}} \mathcal{A}_{k}-a_{1}\left(k, N_{\max }^{k, d}\right) \\
\times a_{2}\left(2,4.5+N_{\max }^{k, d}, 2+N_{\max }^{k, d}, k\right)
\end{gathered}
$$

Proof: The proof follows from (4) and from the equality $\bar{N}_{\mathrm{MT}}^{k, d}=\sum_{e=0}^{+\infty} e q_{e}^{k}=\sum_{e=0}^{N_{\max }^{k, d}-1} e q_{e}^{k}+\sum_{e=N_{\max }^{k, d}}^{+\infty} N_{\max }^{k, d} q_{e}^{k}$. The final result follows by computing the sums in closed-form.

Lemma 5: Consider $\mathcal{A}_{k}=\mathcal{A}_{k}\left(\varepsilon_{d, o}\right)=\mathrm{AP}_{k} / \lambda_{k}^{(\mathrm{A})}$ for $k \in$ $\{\mathrm{M}, \mathrm{S}\}, d \in\{\mathrm{L} 1, \mathrm{~L} 2\}$, and $o \in\{\mathrm{H}, \mathrm{F}\}$. Let $z_{\text {sel }}(\cdot, \cdot)$ be the function defined in Table II. The following identities hold true:

$$
\begin{gathered}
\sum_{e=0}^{t-1} \bar{q}_{e}^{k}+\sum_{e=t}^{+\infty} \frac{t}{e+1} \bar{q}_{e}^{k}=z_{\text {sel }}(k, t) \\
\sum_{e=1}^{+\infty} \frac{1}{e+1} \bar{q}_{e}^{k}=z_{\text {sel }}(k, 1) \\
=\frac{1}{\lambda_{\mathrm{MT}} \mathcal{A}_{k}}\left(1-\left(1+\frac{\lambda_{\mathrm{MT}} \mathcal{A}_{k}}{3.5}\right)^{-3.5}\right)
\end{gathered}
$$

Proof: The proofs follow from (5) and by computing the sums in closed-form.

Remark 4: $z_{\text {sel }}(k, t)$ in (8) can be interpreted as the probability that an MT is selected to use one out of $t$ resources that are available in one cell (among those with at least one MT).

Lemma 6: Let $\mathbf{R}^{d, o}$ for $d \in\{\mathrm{L} 1, \mathrm{~L} 2\}$ and $o \in\{\mathrm{H}, \mathrm{F}\}$ be the $\mathcal{L} \times \mathcal{L}$ matrix of transition probabilities of the discrete Markov chain that describes the temporal dynamics of the batteries of the SBSs. The $(i, j)$ th entry of $\mathbf{R}^{d, o}, r_{i, j}$, is the probability that the battery status moves from level (or state) $i$ to level (or state) $j$. Also, let $\mathbf{v}^{d, o}$ be the $1 \times \mathcal{L}$ vector of steady state probabilities of the discrete Markov chain. The $s$ th entry of $\mathbf{v}^{d, o}, v_{s}^{d, o}$, is the probability that, at the equilibrium, the battery level is $s$. Then, $\mathbf{v}^{d, o}$ is the solution of the following equation [26]:

$$
\mathbf{v}^{d, o} \mathbf{R}^{d, o}\left(\mathbf{v}^{d, o}\right)=\mathbf{v}^{d, o} \quad \text { subject to } \quad \sum_{s=0}^{\mathcal{L}-1} v_{s}^{d, o}=1
$$

Therefore, the probability, $\varepsilon_{d, o}$, that an SBS is unavailable is equal to $\varepsilon_{d, o} \triangleq \sum_{s=0}^{m_{1}^{d}-1} v_{s}^{d, o}$.

Proof: Eq. (9) follows by definition of equilibrium of a discrete Markov chain, and $\varepsilon_{d, o}$ follows by considering that 


$$
\begin{aligned}
& P_{\mathrm{InA}}^{\mathrm{M}, \mathrm{L} 1} \triangleq 1-\frac{\lambda_{\mathrm{MT}} \mathcal{A}_{\mathrm{M}}}{N_{\mathrm{RB}}}+\frac{1}{N_{\mathrm{RB}}} \times \begin{cases}z_{\text {inc }}^{1}\left(\mathrm{M}, N_{\mathrm{RB}}\right) & \text { if } N_{\mathrm{max}}^{\mathrm{M}, \mathrm{L} 1}=N_{\mathrm{RB}} \\
z_{\text {inc }}^{2}\left(\mathrm{M}, N_{\mathrm{Load}}^{\mathrm{M}}\right) & \text { if } N_{\mathrm{max}}^{\mathrm{M}, \mathrm{L} 1}=N_{\mathrm{Load}}^{\mathrm{M}}\end{cases} \\
& P_{\mathrm{InA}}^{\mathrm{S}, \mathrm{L} 1} \triangleq \sum_{N_{\mathrm{MT}}=1}^{N_{\max }^{\mathrm{S}, \mathrm{L} 1}-1}\left(1-\frac{\lambda_{\mathrm{MT}} \mathcal{A}_{\mathrm{S}}}{N_{\mathrm{RB}}}+\frac{z_{\mathrm{inc}}^{2}\left(\mathrm{~S}, N_{\mathrm{MT}}\right)}{N_{\mathrm{RB}}}\right) \frac{f_{1}\left(\mathrm{~L} 1, o, N_{\mathrm{MT}}\right)}{1-\varepsilon_{\mathrm{L} 1, o}}+\frac{f_{2}\left(\mathrm{~L} 1, o, N_{\max }^{\mathrm{S}, \mathrm{L} 1}\right)}{1-\varepsilon_{\mathrm{L} 1, o}} \\
& \times\left(1-\frac{\lambda_{\mathrm{MT}} \mathcal{A}_{\mathrm{S}}}{N_{\mathrm{RB}}}+\frac{1}{N_{\mathrm{RB}}} \times\left\{\begin{array}{ll}
z_{\text {inc }}^{1}\left(\mathrm{~S}, N_{\mathrm{RB}}\right) & \text { if } N_{\max }^{\mathrm{S}, \mathrm{L} 1}=N_{\mathrm{RB}} \\
z_{\text {inc }}^{2}\left(\mathrm{~S}, N_{\mathrm{LB}}\right) & \text { if } N_{\max }^{\mathrm{S}, \mathrm{L} 1}=N_{\mathrm{LB}}
\end{array}\right)\right.
\end{aligned}
$$

an SBS is available if its battery level is greater than $m_{1}^{d}$.

Remark 5: In contrast to conventional formulations of the steady state equation in (9) (see, e.g., [7]), our case study is different. The matrix of transition probabilities depends, in particular, on the vector of steady state probabilities itself, which makes the calculation of $\mathbf{v}^{d, o}$, and, therefore, of $\varepsilon_{d, o}$, unlikely in closed-form. Further details, the closed-form expression of $\mathbf{R}^{d, o}$, and some examples to understand how to construct it are given in Section [III-C] In spite of this analytical intractability, we show, in the sequel, that relevant key performance indicators for the network model under analysis can be formulated, given $\varepsilon_{d, o}$, in closed-form. Therefore, the proposed method provides one with a semi-analytical approach that is shown to be more efficient compared with Monte Carlo simulations. Semi-analytical approaches have been proposed in other papers as well. In [12], e.g., the term semi-analytical is referred to combining, in a simplified system model, analysis with Monte Carlo simulations. Our approach is, on the other hand, different. In 9), in fact, all the functions and parameters are available in closed-form. Therefore, the term semi-analytical is referred to the need of solving (9) numerically, but without the need of using Monte Carlo simulation methods. In Section V, in addition, we prove that our approach provides, in simplified case studies, insights for system design and guidelines for system optimization.

Remark 6: In 9, we assume that the temporal evolution of the batteries employed for energy harvesting at the SBSs is statistically equal. This is possible because (4)-(8) are averaged over the distribution of the area of the cells [24]. As detailed in Remark 2, this is an approximation.

The following two propositions yield the inactive probability of the MBSs and SBSs. Under L1, the inactive probability is defined as the probability that a BS does not transmit on a randomly chosen RB. Under L2, the inactive probability is defined as the probability that a BS is not in transmission mode. In the latter case, as discussed in the previous sections, an inactive MBS is in idle mode, and an inactive SBS is in energy harvesting mode. Three lemmas are, however, first reported, since they are used to prove the two following propositions. All the auxiliary functions used in the following lemmas and propositions are defined in Table II

Lemma 7: Consider an available SBS. The probability that it has sufficient power to serve exactly $N_{\mathrm{MT}} \geq 1$ MTs can be formulated, for $d \in\{\mathrm{L} 1, \mathrm{~L} 2\}$ and $o \in\{\mathrm{H}, \mathrm{F}\}$, as follows:

$$
\begin{aligned}
& P_{\text {serve }}^{\mathrm{S}}\left(d, o, N_{\mathrm{MT}}\right) \\
& \triangleq \frac{1}{1-\varepsilon_{d, o}} \times\left\{\begin{array}{lll}
f_{1}\left(d, o, N_{\mathrm{MT}}\right) & \text { if } & N_{\mathrm{MT}}<N_{\max }^{\mathrm{S}, d} \\
f_{2}\left(d, o, N_{\max }^{\mathrm{S}, d}\right) & \text { if } & N_{\mathrm{MT}}=N_{\max }^{\mathrm{S}, d}
\end{array}\right.
\end{aligned}
$$

Proof: See Appendix A.

Lemma 8: Consider an MBS or an available SBS that has sufficient power to serve exactly $1 \leq N_{\mathrm{MT}} \leq N_{\max }^{k, d}$ MTs. Under load model L1, the probability that a BS is inactive on an $\mathrm{RB}$ can be formulated, for $k \in\{\mathrm{M}, \mathrm{S}\}$ and $o \in\{\mathrm{H}, \mathrm{F}\}$, as follows:

$$
\begin{aligned}
& P_{\text {inactive }}^{\mathrm{L} 1}\left(k, o, N_{\mathrm{MT}}\right) \\
& \triangleq \begin{cases}\sum_{n=0}^{N_{\mathrm{RB}}}\left(1-\frac{n}{N_{\mathrm{RB}}}\right) q_{n}^{k} & \text { if } \quad N_{\mathrm{MT}} \geq N_{\mathrm{RB}} \\
\sum_{n=0}^{N_{\mathrm{MT}}-1}\left(1-\frac{n}{N_{\mathrm{RB}}}\right) q_{n}^{k} & \\
+\sum_{n=N_{\mathrm{MT}}}^{+\infty}\left(1-\frac{N_{\mathrm{MT}}}{N_{\mathrm{RB}}}\right) q_{n}^{k} & \text { if } \quad N_{\mathrm{MT}}<N_{\mathrm{RB}}\end{cases}
\end{aligned}
$$

Proof: The proof follows by applying the total probability theorem as a function of the number of MTs associated to a BS, and by taking into account that the probability of transmitting on an RB is equal to the ratio between the number of MTs in the cell and the number of RBs.

Lemma 9: The notable identities hold true $\left(\mathcal{A}_{k}=\mathcal{A}_{k}\left(\varepsilon_{\mathrm{L} 1, o}\right)\right.$ for $k \in\{\mathrm{M}, \mathrm{S}\}$ and $o \in\{\mathrm{H}, \mathrm{F}\}$ ):

$$
\begin{aligned}
\mathcal{I}_{\mathrm{RB}}\left(k, o, N_{\mathrm{RB}}\right) & \triangleq \sum_{n=0}^{N_{\mathrm{RB}}}\left(1-\frac{n}{N_{\mathrm{RB}}}\right) q_{n}^{k} \\
& =1-\frac{\lambda_{\mathrm{MT}} \mathcal{A}_{k}}{N_{\mathrm{RB}}}+\frac{z_{\mathrm{inc}}^{1}\left(k, N_{\mathrm{RB}}\right)}{N_{\mathrm{RB}}} \\
\mathcal{I}_{\mathrm{MT}}\left(k, o, N_{\mathrm{MT}}\right) & \triangleq \sum_{n=0}^{N_{\mathrm{MT}}-1}\left(1-\frac{n}{N_{\mathrm{RB}}}\right) q_{n}^{k} \\
& +\sum_{n=N_{\mathrm{MT}}}^{+\infty}\left(1-\frac{N_{\mathrm{MT}}}{N_{\mathrm{RB}}}\right) q_{n}^{k} \\
& =1-\frac{\lambda_{\mathrm{MT}} \mathcal{A}_{k}}{N_{\mathrm{RB}}}+\frac{z_{\mathrm{inc}}^{2}\left(k, N_{\mathrm{MT}}\right)}{N_{\mathrm{RB}}}
\end{aligned}
$$

Proof: The proof follows by inserting the analytical expression of $q_{n}^{k}$ in (4) into (12) and (13), and by subsequently computing the resulting sums in closed-form.

Proposition 1: Define $N_{\mathrm{LB}} \triangleq \min \left\{N_{\text {Load }}^{\mathrm{S}}, N_{\text {Bat }}^{\mathrm{L} 1}\right\}$. Under 
TABLE III: Examples of matrices $\left.\mathbf{R}^{d, o}\left(J_{b}=1-\sum_{i=0}^{b} p_{i}\right): \mathcal{L}=5, m_{1}^{\mathrm{L} 1}=2, m^{\mathrm{L} 1}=2, m_{1}^{\mathrm{L} 2}=3, m^{\mathrm{L} 2}=1\right)$

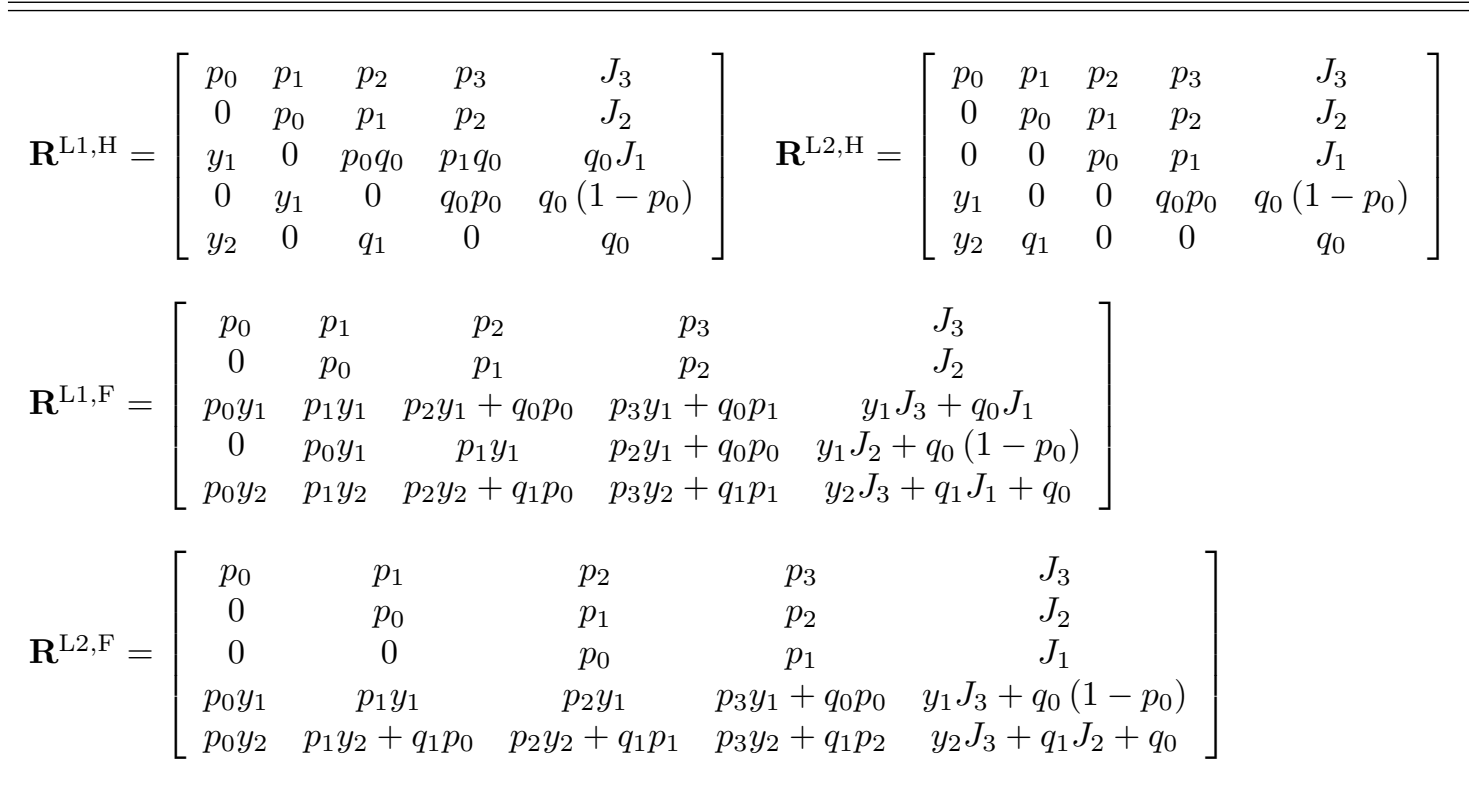

load model L1, the probability that an MBS and an SBS are inactive is available in (14) and (15) at the top of the previous page, respectively, where $\mathcal{A}_{k}=\mathcal{A}_{k}\left(\varepsilon_{\mathrm{L} 1, o}\right)$ for $k \in\{\mathrm{M}, \mathrm{S}\}$ and $o \in\{\mathrm{H}, \mathrm{F}\}$.

Proof: See Appendix B.

Proposition 2: Under load model L2, the probability that an MBS and an SBS are inactive can be formulated as follows $\left(\mathcal{A}_{k}=\mathcal{A}_{k}\left(\varepsilon_{\mathrm{L} 2, o}\right)\right.$ for $k \in\{\mathrm{M}, \mathrm{S}\}$ and $\left.o \in\{\mathrm{H}, \mathrm{F}\}\right)$ :

$$
P_{\mathrm{InA}}^{k, \mathrm{~L} 2} \triangleq\left(1+\frac{\lambda_{\mathrm{MT}} \mathcal{A}_{k}}{3.5}\right)^{-3.5}
$$

Proof: Under load model L2, the MBSs and SBSs are inactive if no MT is associated to them. Then, the inactive probability is equal to $q_{e}^{k}$ in (4) by setting $e=0$.

Remark 7: In general, $P_{\mathrm{InA}}^{k, \mathrm{~A} 1} \neq P_{\mathrm{InA}}^{k, \mathrm{~L} 2}$. If $N_{\mathrm{RB}}=1$ or $\mathcal{L}=2$, i.e., the battery is only empty or full (two-state model), it can be verified by direct inspection of Proposition 1 that $P_{\mathrm{InA}}^{k, \mathrm{~L} 1}=P_{\mathrm{InA}}^{k, \mathrm{~L} 2}$.

\section{Discrete Markov Chain - Matrix of Transition Probabilities}

In this section, based on the considered system model and the proposed approximations for performance analysis, we provide explicit closed-form expressions of the matrix of transition probabilities for the two considered load models ( $d \in\{\mathrm{L} 1, \mathrm{~L} 2\}$ ) and for half-duplex and full-duplex energy harvesting modes $(o \in\{\mathrm{H}, \mathrm{F}\})$. Before introducing the general expression of $\mathbf{R}^{d, o}$ in $(9)$, we illustrate some toy examples in order to understand how $\mathbf{R}^{d, o}$ is constructed. The four examples of matrices for $d \in\{\mathrm{L} 1, \mathrm{~L} 2\}$ and $o \in\{\mathrm{H}, \mathrm{F}\}$ are reported in Table III They are obtained by considering the following setup: $\mathcal{L}=5, \widetilde{P}_{\text {cir }}^{\mathrm{S}}=1, \widetilde{P}_{\text {tx }}^{\mathrm{S}}$,tot $=2$, and $N_{\mathrm{RB}}=2$. Therefore, $m_{1}^{\mathrm{L} 1}=2, m^{\mathrm{L} 1}=2, m_{1}^{\mathrm{L} 2}=3$, and $m^{\mathrm{L} 2}=1$. For notational simplicity, since only the SBSs are of interest in this section, we employ the simplified notation $q_{e}^{\mathrm{S}}=q_{e}$ and $y_{e}^{\mathrm{S}}=y_{e}$.
The matrices in Table III are obtained based on the following considerations. The first two rows of $\mathbf{R}^{\mathrm{L} 1, \mathrm{H}}$ and $\mathbf{R}^{\mathrm{L} 1, \mathrm{~F}}$ are the same, since the SBS has no sufficient power to serve any MTs if it is either in state $s=0$ or in state $s=1$. If the battery is in state $s=2$, then the SBS can serve at most one MT depending on the current battery state and the presence of MTs in its cell. The operation of an SBS equipped with a half-duplex and a full-duplex battery is, however, different. As an example, let us consider $r_{3,3}$. In half-duplex mode, a battery in state $s=3$ continues to stay in state $s=3$ if there are no MTs in its cell (whose probability is $q_{0}$ ) and, simultaneously, no power packets are harvested (whose probability is $p_{0}$ ). In full-duplex mode, on the other hand, $r_{3,3}$ is the sum of the probabilities of two incompatible events: (i) there is at least one MT in the cell (whose probability is $y_{1}$ ) and, simultaneously, two power packets are harvested (whose probability is $p_{2}$ ) or (ii) there are no MTs in the cell (whose probability is $q_{0}$ ) and, simultaneously, no power packets are harvested (whose probability is $p_{0}$ ). Similar considerations can be made for $\mathbf{R}^{\mathrm{L} 2, \mathrm{H}}$ and $\mathbf{R}^{\mathrm{L} 2, \mathrm{~F}}$. In this case, however, the SBS has no sufficient power to serve any MTs if it is in states $s=0, s=1$, and $s=2$. This is due to the different power consumption of the SBS under load models L1 and L2. It is apparent, therefore, that both the load model and the energy harvesting duplexing scheme determine the steady state regime of the batteries, and, therefore, the probability that an SBS is available, i.e., $1-\varepsilon_{d, o}$.

By using the same line of thought as for the examples in Table III the general formulation of the matrices of transition probabilities, $\mathbf{R}^{d, o}$, is reported in Table IV and Table $\mathrm{V}$ for half-duplex and full-duplex operating modes, respectively. In both tables, $\mathbf{R}_{i} \triangleq\left[\begin{array}{llll}r_{i, 1} & r_{i, 2} & \ldots & r_{i, \mathcal{L}}\end{array}\right]$ denotes the $i$ th row of $\mathbf{R}^{d, o}$ and $\mathbf{R}_{i}^{-} \triangleq\left[\begin{array}{llll}r_{i, 2} & r_{i, 3} & \ldots & r_{i, \mathcal{L}-2}\end{array}\right]$ is a reduced version of $\mathbf{R}_{i}$, which has $\mathcal{L}-2$ elements. Similar to Table III the notation $q_{e}^{\mathrm{S}}=q_{e}$ and $y_{e}^{\mathrm{S}}=y_{e}$ is used in Tables IV and $\mathrm{V}$ 
TABLE IV: Algorithm for computing the matrix of transition probabilities - Half-duplex mode

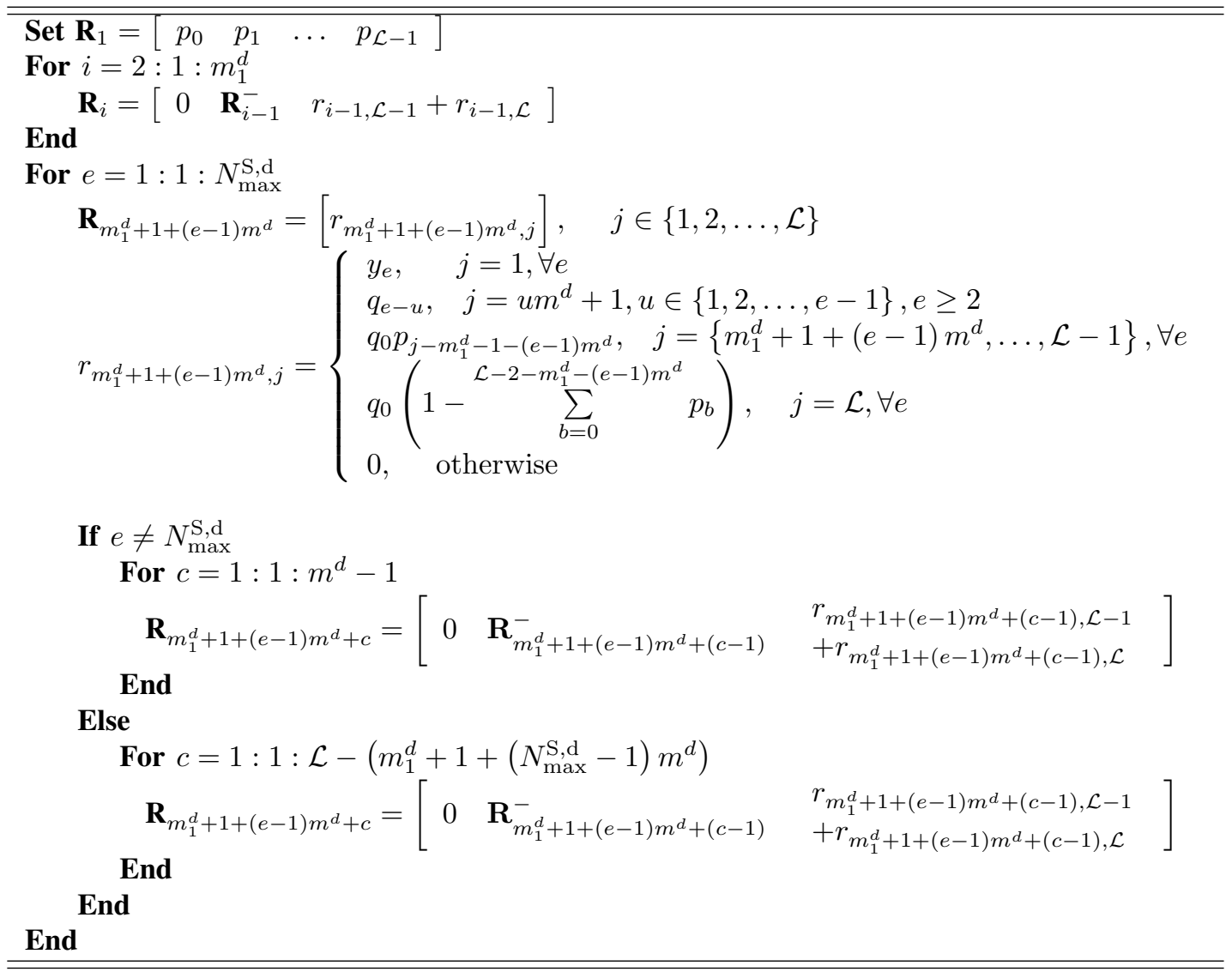

TABLE V: Algorithm for computing the matrix of transition probabilities - Full-duplex mode

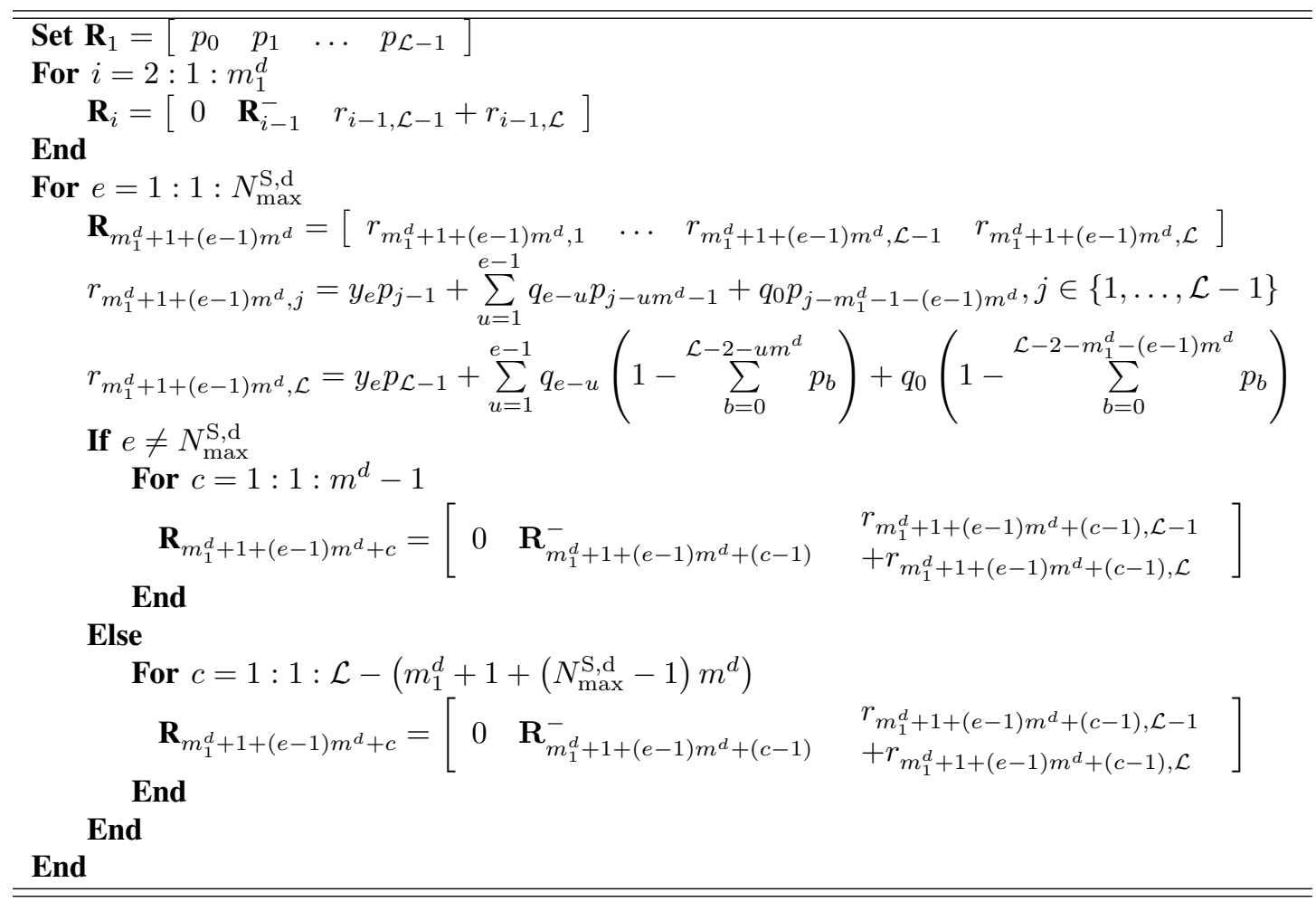




$$
\operatorname{SIR}_{k, d} \triangleq \frac{P_{\mathrm{tx}, \mathrm{tot}}^{k} g^{(0)} / K_{k}^{(0)}}{\sum_{i \in \Psi_{k}^{(\mathrm{A}, \mathrm{I}), d}} \frac{P_{\mathrm{tx}, \mathrm{tot}}^{k} g_{i}}{l_{k}\left(r_{i}\right)} \mathbf{1}\left(l_{k}\left(r_{i}\right)>K_{k}^{(0)}\right)+\sum_{i \in \Psi_{\tilde{k}}^{(\mathrm{A}, \mathrm{I}), d}} \frac{P_{\mathrm{tx}, \mathrm{tot}}^{\tilde{\tilde{k}}} g_{i}}{l_{\tilde{k}}\left(r_{i}\right)} \mathbf{1}\left(l_{\tilde{k}}\left(r_{i}\right)>\frac{T_{k} P_{\mathrm{tx}, \mathrm{tot}}^{\tilde{z}}}{T_{\tilde{k}} P_{\mathrm{tx}, \mathrm{tot}}^{k}} K_{k}^{(0)}\right)}
$$

\section{Performance Analysis}

In this section, we introduce closed-form expressions, given $\varepsilon_{d, o}$, of the coverage probability, the spectral efficiency, the power consumption, and the EE of the cellular network model under analysis. These key performance indicators are computed based on the definition of coverage probability recently introduced in [1]. Similar to [1], our analysis relies on the approximation that, in each tier, the set of interfering BSs is an independently thinned version of the set of available BSs whose retaining probability is the probability that a BS is active, which depends on the tier, the load model, and the energy harvesting duplexing mode. The energy harvesting duplexing mode is implicitly taken into account in the formulation of the Markov chain, and, therefore, in $\varepsilon_{d, o}$. For these reasons, unless necessary, it is omitted from our notation. The proposed modeling approximation can be formalized as follows.

Approximation 3: Let $\Psi_{k}^{(\mathrm{A})}$ be the PPP of available BSs in tier $k \in\{\mathrm{M}, \mathrm{S}\}$ whose density is $\lambda_{k}^{(\mathrm{A})}$. The set of interfering BSs in the $k$ th tier is a PPP, denoted by $\Psi_{k}^{(\mathrm{A}, \mathrm{I}), d}$, whose density is $\lambda_{k}^{(\mathrm{A}, \mathrm{I}), d} \triangleq \lambda_{k}^{(\mathrm{A})}\left(1-P_{\mathrm{InA}}^{k, d}\right)$ for $k \in\{\mathrm{M}, \mathrm{S}\}$ and $d \in\{\mathrm{L} 1, \mathrm{~L} 2\}$.

From [1], the coverage probability of a selected MT that is associated to an available BS is defined as $\mathrm{P}_{d}^{(\operatorname{cov})}\left(\gamma_{\mathrm{D}}, \gamma_{\mathrm{A}}\right) \triangleq$ $\sum_{k \in\{\mathrm{M}, \mathrm{S}\}} \mathrm{P}_{k, d}^{(\text {cov })}\left(\gamma_{\mathrm{D}}, \gamma_{\mathrm{A}}\right)$, where $(k \neq \tilde{k} \in\{\mathrm{M}, \mathrm{S}\})$ :

$$
\begin{aligned}
& \mathrm{P}_{k, d}^{(\text {cov })}\left(\gamma_{\mathrm{D}}, \gamma_{\mathrm{A}}\right) \\
& \triangleq \operatorname{Pr}\left\{\operatorname{SIR}_{k, d} \geq \gamma_{\mathrm{D}}, \mathrm{ASNR}_{k, d} \geq \gamma_{\mathrm{A}}, \frac{T_{\widetilde{k}} K_{\widetilde{k}}^{(0)}}{P_{\mathrm{tx}, \text { tot }}} \geq \frac{T_{k} K_{k}^{(0)}}{P_{\mathrm{tx}, \mathrm{tot}}^{k}}\right\}
\end{aligned}
$$

and $\gamma_{\mathrm{D}}$ is the decoding threshold during data transmission, $\gamma_{\mathrm{A}}$ is the detection threshold during cell association, $\mathrm{ASNR}_{k, d} \triangleq P_{\mathrm{tx}, \text { tot }}^{k} / K_{k}^{(0)} \sigma_{\mathrm{N}}^{2}$ is the Average Signal-to-Noise Ratio (ASNR), and the Signal-to-Interference Ratio (SIR) is defined in (18) shown at the top of this page.

Proposition 3: The coverage probability in (17) can be formulated as follows:

$$
\begin{aligned}
\mathrm{P}_{k, d}^{(\text {cov })}\left(\gamma_{\mathrm{D}}, \gamma_{\mathrm{A}}\right) & \\
= & \frac{1-\exp \left(-\pi \lambda_{k}^{(\mathrm{A})}\left(P_{\text {tx }, \text { tot }}^{k} /\left(\kappa \gamma_{\mathrm{A}} \sigma_{\mathrm{N}}^{2}\right)\right)^{2 / \beta} \mathcal{D}_{k, \tilde{k}}\right)}{\mathcal{D}_{k, \tilde{k}}}
\end{aligned}
$$

where $k \neq \tilde{k} \in\{\mathrm{M}, \mathrm{S}\}$ and the following shorthand notation is used:

$$
\begin{aligned}
\mathcal{D}_{k, \tilde{k}} \triangleq & 1+\frac{\lambda_{\tilde{k}}^{(\mathrm{A})}}{\lambda_{k}^{(\mathrm{A})}}\left(\frac{T_{k} P_{\mathrm{tx}, \mathrm{tot}}^{\tilde{k}}}{T_{\tilde{k}} P_{\mathrm{tx}, \mathrm{tot}}^{k}}\right)^{2 / \beta}+\frac{\lambda_{k}^{(\mathrm{A}, \mathrm{I}), d}}{\lambda_{k}^{(\mathrm{A})}} c\left(\gamma_{\mathrm{D}}\right) \\
& +\frac{\lambda_{\tilde{k}}^{(\mathrm{A}, \mathrm{I}), d}}{\lambda_{k}^{(\mathrm{A})}}\left(\frac{T_{k} P_{\mathrm{tx}, \mathrm{tot}}^{\tilde{k}}}{T_{\tilde{k}} P_{\mathrm{tx}, \mathrm{tot}}^{k}}\right)^{2 / \beta} c\left(\gamma_{\mathrm{D}} \frac{T_{\tilde{k}}}{T_{k}}\right)
\end{aligned}
$$

Proof: The proof follows by using similar steps as those in [1, Appendix A], which are generalized to a two-tier system model by using the same approach as in [23, Section IV].

Remark 8: In contrast with [1, Remark 9], the coverage probability in 19) depends on the number of MTs in the cell, and the power level of the batteries of the SBSs. Other differences compared with [1] are the following: (i) under L1, multiple RBs are considered, and, thus, multiple MTs can be scheduled in each cell; (ii) under L1 and L2, there exists a maximum number of MTs that can be scheduled in each cell, i.e., $N_{\text {Load }}^{k}$ in the $k$ th tier; and (iii) the finite capacity of the batteries and the availability of sufficient energy are taken into account.

Before formulating the general expression of the Potential Spectral Efficiency (PSE) [1], we report four lemmas that yield the PSE by conditioning on the serving BS (MBS and SBS) and the load model (L1 and L2). Each case study is, in fact, different and deserves attention.

Lemma 10: Let the serving BS be an MBS. Under load model L1, the PSE can be formulated as follows:

$$
\begin{aligned}
\mathrm{PSE}_{\mathrm{M}, \mathrm{L} 1} & \triangleq \lambda_{\mathrm{MT}}\left(\mathrm{BW}_{\text {tot }} / N_{\mathrm{RB}}\right) \log _{2}\left(1+\gamma_{\mathrm{D}}\right) \\
& \times z_{\text {sel }}\left(\mathrm{M}, N_{\max }^{\mathrm{M}, \mathrm{L} 1}\right) \mathrm{P}_{\mathrm{M}, \mathrm{L} 1}^{(\mathrm{cov})}\left(\gamma_{\mathrm{D}}, \gamma_{\mathrm{A}}\right)
\end{aligned}
$$

Proof: See Appendix C

Lemma 11: Let the serving BS be an MBS. Under load model L2, the PSE can be formulated as follows:

$$
\begin{aligned}
\mathrm{PSE}_{\mathrm{M}, \mathrm{L} 2} & \triangleq \lambda_{\mathrm{MT}} \mathrm{BW}_{\mathrm{tot}} \log _{2}\left(1+\gamma_{\mathrm{D}}\right) \\
& \times z_{\mathrm{sel}}(\mathrm{M}, 1) \mathrm{P}_{\mathrm{M}, \mathrm{L} 2}^{(\mathrm{cov})}\left(\gamma_{\mathrm{D}}, \gamma_{\mathrm{A}}\right)
\end{aligned}
$$

Proof: See Appendix C

Lemma 12: Let the serving BS be an SBS. Under load model L1, the PSE can be formulated as follows:

$$
\begin{aligned}
\mathrm{PSE}_{\mathrm{S}, \mathrm{L} 1} & \triangleq \lambda_{\mathrm{MT}}\left(\mathrm{BW}_{\text {tot }} / N_{\mathrm{RB}}\right) \log _{2}\left(1+\gamma_{\mathrm{D}}\right) \\
& \times z_{\text {sel, serve }}\left(\mathrm{S}, N_{\max }^{\mathrm{S}, \mathrm{L} 1}\right) \mathrm{P}_{\mathrm{S}, \mathrm{L} 1}^{(\operatorname{cov})}\left(\gamma_{\mathrm{D}}, \gamma_{\mathrm{A}}\right)
\end{aligned}
$$

where the shorthand notation $z_{\mathrm{sel}, \text { serve }}\left(\mathrm{S}, N_{\max }^{\mathrm{S}, \mathrm{L} 1}\right)=$ $\sum_{N_{\mathrm{MT}}=1}^{N_{\text {max }}^{\mathrm{S}, \mathrm{LL}}} z_{\text {sel }}\left(\mathrm{S}, N_{\mathrm{MT}}\right) P_{\text {serve }}^{\mathrm{S}}\left(\mathrm{L} 1, o, N_{\mathrm{MT}}\right)$ is used.

Proof: See Appendix C

Lemma 13: Let the serving BS be an SBS. Under load model $\mathrm{L} 2$, the PSE can be formulated as follows:

$$
\begin{aligned}
\mathrm{PSE}_{\mathrm{S}, \mathrm{L} 2} & \triangleq \lambda_{\mathrm{MT}} \log _{2}\left(1+\gamma_{\mathrm{D}}\right) \mathrm{BW}_{\text {tot }} \\
& \times z_{\text {sel }}(\mathrm{S}, 1) \mathrm{P}_{\mathrm{S}, \mathrm{L} 2}^{(\text {cov }}\left(\gamma_{\mathrm{D}}, \gamma_{\mathrm{A}}\right)
\end{aligned}
$$

Proof: See Appendix C

Remark 9: It may seem counterintuitive that (24) is independent of $P_{\text {serve }}^{\mathrm{S}}(\cdot)$, i.e., the probability of serving some MTs as a function of the power level of the battery. This originates from the uniform allocation strategy of the resources among 
the MTs, as the proof in Appendix C elucidates. It is worth mentioning that 24) depends on the amount of harvested energy through the probability $\varepsilon_{d, o}$, and on the storage capacity of the battery through $N_{\max }^{\mathrm{S}, \mathrm{L} 1}$.

Proposition 4: The PSE under load model $d \in\{\mathrm{L} 1, \mathrm{~L} 2\}$ can be formulated as follows:

$$
\mathrm{PSE}_{d} \triangleq \mathrm{PSE}_{\mathrm{M}, d}+\mathrm{PSE}_{\mathrm{S}, d}
$$

Proof: The proof follows from Lemmas 10 by using the total probability theorem.

We close this section by formulating the EE. We note that the network power consumption depends only on the MBSs, since the SBSs are powered only through renewable energy sources. So, there is no power expenditure for the power grid.

Proposition 5: The EE (bit/Joule) under load model $d \in$ $\{\mathrm{L} 1, \mathrm{~L} 2\}$ can be formulated as $\mathrm{EE}_{d} \triangleq \mathrm{PSE}_{d} / \mathrm{P}_{d}^{\text {(grid) }}$, where $\mathrm{P}_{d}^{\text {(grid) }}$ is the power dissipated (Watt / unit area) by the MBSs:

$$
\begin{aligned}
\mathrm{P}_{\mathrm{L} 1}^{(\text {grid })} & \triangleq \lambda_{\mathrm{M}}^{(\mathrm{A})} N_{\mathrm{RB}}\left(P_{\text {circ }}^{\mathrm{M}}+P_{\mathrm{tx}, \mathrm{tot}}^{\mathrm{M}} / N_{\mathrm{RB}}\right)\left(1-P_{\mathrm{InA}}^{\mathrm{M}, \mathrm{L} 1}\right) \\
& +\lambda_{\mathrm{M}}^{(\mathrm{A})} P_{\text {idle }} q_{0}^{\mathrm{M}} \\
\mathrm{P}_{\mathrm{L} 2}^{(\text {grid })} & \triangleq \lambda_{\mathrm{M}}^{(\mathrm{A})} P_{\mathrm{tx}, \text { tot }}^{\mathrm{M}}\left(1-q_{0}^{\mathrm{M}}\right) \\
& +\lambda_{\mathrm{M}}^{(\mathrm{A})} P_{\text {idle }} q_{0}^{\mathrm{M}}+\lambda_{\mathrm{M}}^{(\mathrm{A})} P_{\text {circ }}^{\mathrm{M}} \bar{N}_{\mathrm{MT}}^{\mathrm{M}, \mathrm{L} 2}
\end{aligned}
$$

where $\bar{N}_{\mathrm{MT}}^{\mathrm{M}, \mathrm{L} 2}$ is defined in (6), and $q_{0}^{\mathrm{M}}=$ $\left(1+\lambda_{\mathrm{MT}} \mathcal{A}_{\mathrm{M}} / 3.5\right)^{-3.5}$, which denotes the probability that there are no MTs inside a cell, follows from (4) by setting $e=0$.

Proof: The proof follows by taking into account the power consumption of the MBSs when they operate in transmission and idle modes, according to the probability of occurrence of these two operating regimes. The term $\bar{N}_{\mathrm{MT}}^{\mathrm{M}, \mathrm{L} 2}$ accounts for the fact that, under load model L2, the circuits power consumption depends on the (random) number of MTs inside the cells.

\section{Optimization OF THE ENERGy EFFICIENCY - A Few Case Studies}

The EE in Proposition 5 is formulated, given $\varepsilon_{d, o}$, in closedform. For its computation, in particular, only the steady state probabilities of the discrete Markov chain that models the temporal dynamics of the batteries of the SBSs need to be obtained numerically. The computation of the EE is, therefore, simple and efficient. The need, however, of computing the steady state probabilities numerically makes it difficult to identify important structural properties of the $\mathrm{EE}$ as a function of the many system parameters. An important question is, for example, whether there exists an optimal density of the BSs that maximizes the EE [1]. To shed light on the EE in the presence of renewable energy sources and to gain relevant engineering insights for optimizing the network deployment, we consider a few relevant case studies in the next subsections.

\section{A. Optimization as a Function of the Transmit Power of the Base Stations}

In this section, we optimize the $\mathrm{EE}$ as a function of the transmit power of the BSs. For simplicity, we consider
$P_{\mathrm{tx}, \mathrm{tot}}^{\mathrm{M}} \triangleq v_{\mathrm{M}} P_{\mathrm{tx}, \text { tot }}$ and $P_{\mathrm{tx}, \text { tot }}^{\mathrm{S}} \triangleq v_{\mathrm{S}} P_{\mathrm{tx}, \mathrm{tot}}$, where $v_{\mathrm{M}}$ and $v_{\mathrm{S}}$ are positive constants. We are interested in the following optimization problem:

$$
\max _{P_{\mathrm{tx}, \mathrm{tot}} \in\left[P_{\mathrm{tx}, \mathrm{tot}}^{\min }, P_{\mathrm{tx}, \mathrm{tot}}^{\max }\right]}\left\{\mathrm{EE}_{d}\left(P_{\mathrm{tx}, \mathrm{tot}}\right)=\frac{\mathrm{PSE}_{d}\left(P_{\mathrm{tx}, \mathrm{tot}}\right)}{\mathrm{P}_{d}^{\text {(grid })}\left(P_{\mathrm{tx}, \mathrm{tot}}\right)}\right\}
$$

where $P_{\mathrm{tx}, \mathrm{tot}}^{\min }$ and $P_{\mathrm{tx}, \mathrm{tot}}^{\max }$ are the minimum and maximum values that are allowed for $P_{\mathrm{tx}, \mathrm{tot}}$.

The solution of $\mathcal{P}_{P_{\mathrm{tx}, \text { tot }}}$ in 28 is characterized in the following theorem.

Theorem 1: The EE in 28 is a unimodal and strictly pseudo-concave function in $P_{\mathrm{tx}, \text { tot }}$.

Proof: By inspection of the EE in Proposition 5. we note that the PSE can be written as $\operatorname{PSE}_{d}\left(P_{\mathrm{tx}, \mathrm{tot}}\right) \propto \bar{a}_{1}\left(1-\exp \left(-\bar{b}_{1}\left(P_{\mathrm{tx}, \mathrm{tot}}\right)^{2 / \beta}\right)\right)+$ $\bar{a}_{2}\left(1-\exp \left(-\bar{b}_{2}\left(P_{\text {tx }, \text { tot }}\right)^{2 / \beta}\right)\right)$, where $\bar{a}_{1}, \bar{a}_{2}, \bar{b}_{1}$, and $\bar{b}_{2}$ are positive constant terms that are independent of $P_{\mathrm{tx}, \mathrm{tot}}$. By computing the second-order derivative, it can be proved that both addends are strictly concave functions in $P_{\mathrm{tx}, \mathrm{tot}}$ for $\beta>2$. The PSE is, therefore, a strictly concave function in $P_{\text {tx tot }}$, since it is the sum of two strictly concave functions. Also, the power consumption can be written as $\mathrm{P}_{d}^{\text {(grid) }}\left(P_{\mathrm{tx}, \mathrm{tot}}\right)=\bar{d}_{1} P_{\mathrm{tx}, \mathrm{tot}}+\bar{d}_{2}$, where $\bar{d}_{1}$ and $\bar{d}_{2}$ are positive constant terms that are independent of $P_{\mathrm{tx}, \text { tot }}$, i.e., $\mathrm{P}_{d}^{\text {(grid) }}$ is a linear function in $P_{\mathrm{tx}, \mathrm{tot}}$. Thus, the EE is the ratio of a strictly concave function and a linear function, i.e., it is strictly pseudo-concave in $\mathcal{P}_{P_{\mathrm{tx}, \text { tot }}}$ and has a single maximizer.

Theorem 1 ensures that there exists a unique value of the transmit power of the BSs that maximizes the EE, and it is the zero of the first-order derivative of the EE in Proposition 5

\section{B. Optimization as a Function of the Density of the Base Stations}

In this section, we optimize the EE as a function of the density of the BSs. This is not an easy task to solve under general operating conditions, and, in particular, for an arbitrary number of power levels of the batteries of the SBSs. To gain relevant engineering insights, therefore, we analyze a simplified but realistic case study. We consider, in particular, load model L1 where a single MT is scheduled for transmission, i.e., $N_{\max }^{k, \mathrm{~L} 1}=1$, and assume that the batteries of the SBSs have only two power levels (states), i.e., $\mathcal{L}=2$, which implies that the batteries can be either empty or fully charged. In addition, we assume $T_{\mathrm{M}}=T_{\mathrm{S}}=1$ and $P_{\mathrm{tx}, \mathrm{tot}}^{\mathrm{M}}=P_{\mathrm{tx}, \mathrm{tot}}^{\mathrm{S}}=P_{\mathrm{tx}, \mathrm{tot}}$.

For ease of writing, we introduce the simplified notation as follows $(o \in\{\mathrm{H}, \mathrm{F}\})$ :

$$
\begin{aligned}
& \varepsilon \triangleq \varepsilon_{\mathrm{L} 1, o} \\
& c \triangleq c\left(\gamma_{\mathrm{D}}\right) \\
& w \triangleq\left(P_{\mathrm{tx}, \mathrm{tot}} /\left(\kappa \gamma_{\mathrm{A}} \sigma_{\mathrm{N}}^{2}\right)\right)^{2 / \beta} \\
& \Delta P \triangleq P_{\mathrm{tx}, \mathrm{tot}}+P_{\text {circ }}-P_{\text {idle }}
\end{aligned}
$$




$$
\begin{aligned}
& \mathcal{G} \triangleq \alpha_{\mathrm{M}}+\alpha_{\mathrm{S}}(1-\varepsilon) \\
& \mathcal{F} \triangleq 1-\left(1+\frac{1}{3.5} \frac{\lambda_{\mathrm{MT}}}{\lambda_{\mathrm{BS}}} \frac{1}{\mathcal{G}}\right)^{-3.5} \\
& \mathcal{Q} \triangleq 1-\exp \left(-\pi w \lambda_{\mathrm{BS}} \mathcal{G}(1+c \mathcal{F})\right)
\end{aligned}
$$

In the considered case study, the EE can be formulated as follows:

$$
\begin{aligned}
\mathrm{EE}_{\mathrm{L} 1} & \triangleq \mathrm{EE} \\
& =\mathrm{BW}_{\text {tot }} \log _{2}\left(1+\gamma_{\mathrm{D}}\right) \frac{\lambda_{\mathrm{BS}} \mathcal{F} \mathcal{G} \mathcal{Q} /(1+c \mathcal{F})}{\lambda_{\mathrm{BS}} \alpha_{\mathrm{M}}\left(\Delta P \mathcal{F}+P_{\text {idle }}\right)}
\end{aligned}
$$

where $\varepsilon$ is the solution of the steady state equation in (9), which, for $o \in\{\mathrm{H}, \mathrm{F}\}$, is:

$$
\begin{gathered}
o=\mathrm{F}:\left(1+\frac{1}{3.5} \frac{\lambda_{\mathrm{MT}}}{\lambda_{\mathrm{BS}}} \frac{1}{\left(\alpha_{\mathrm{M}}+\alpha_{\mathrm{S}}(1-\varepsilon)\right)}\right)^{-3.5} \\
=\frac{p_{0}-\varepsilon}{p_{0}(1-\varepsilon)} \\
\begin{aligned}
o=\mathrm{H}:\left(1+\frac{1}{3.5} \frac{\lambda_{\mathrm{MT}}}{\lambda_{\mathrm{BS}}}\right. & \left.\frac{1}{\left(\alpha_{\mathrm{M}}+\alpha_{\mathrm{S}}(1-\varepsilon)\right)}\right)^{-3.5} \\
& =1-\frac{\left(1-p_{0}\right) \varepsilon}{1-\varepsilon}
\end{aligned}
\end{gathered}
$$

where the conditions $0 \leq \varepsilon \leq p_{0} \leq 1$ if $o=\mathrm{F}$ and $0 \leq \varepsilon \leq$ $\left(2-p_{0}\right)^{-1} \leq 1$ if $o=\mathrm{H}$, respectively, need to be fulfilled in order to ensure that the solution is feasible.

To elucidate the usefulness of the proposed analytical framework, we are interested in computing, if it exists, an optimal density of BS, $\lambda_{\mathrm{BS}}$, that optimizes the EE. In our study, the other system parameters are kept constant, but more general optimization problems can be formulated and studied. We provide the detailed derivation of our findings for the fullduplex case, while, due to space limitations, we provide only the final result for the half-duplex case. Before going into the details of the analysis, it is interesting to analyze the insights that can be gained, as a function of $\lambda_{\mathrm{BS}}$, by direct inspection of the steady state equation in (37) and (38). As an example, we consider the full-duplex operating mode. The finding is summarized in the following lemma.

Lemma 14: At the equilibrium, the probability that an SBS is available, i.e., $1-\varepsilon$, monotonically increases as the ratio between the densities of the BSs and MTs, $\lambda_{\mathrm{BS}} / \lambda_{\mathrm{MT}}$, increases.

Proof: Eq. 237 can be written as $\lambda_{\mathrm{MT}} / \lambda_{\mathrm{BS}}=$ $\mathcal{S}\left(\varepsilon, p_{0}, \alpha_{\mathrm{M}}, \alpha_{\mathrm{S}}\right)$, where $\mathcal{S}(\cdot)$ does not depend on $\lambda_{\mathrm{BS}}$ and $\lambda_{\mathrm{MT}}$. The proof follows since $\mathcal{S}(\varepsilon, \cdot)$ monotonically increases in $\varepsilon$.

Remark 10: The approach employed in Lemma 14 for the analysis of $\varepsilon$ as a function of $\lambda_{\mathrm{MT}} / \lambda_{\mathrm{BS}}$ can be used to study the impact of other system parameters on $\varepsilon$, e.g., $p_{0}, \alpha_{\mathrm{M}}, \alpha_{\mathrm{S}}$.

1) Full-Duplex Case Study: The optimization problem can be formulated as follows:

$\mathcal{P}_{\lambda_{\mathrm{BS}}}:$

$\max _{\lambda_{\mathrm{BS}} \in\left[\lambda_{\mathrm{BS}}^{\min }, \lambda_{\mathrm{BS}}^{\max }\right]}\left\{\frac{\mathcal{F}\left(\lambda_{\mathrm{BS}}\right) \mathcal{G}\left(\lambda_{\mathrm{BS}}\right) \mathcal{Q}\left(\lambda_{\mathrm{BS}}\right) /\left(1+c \mathcal{F}\left(\lambda_{\mathrm{BS}}\right)\right)}{\left(\Delta P \mathcal{F}\left(\lambda_{\mathrm{BS}}\right)+P_{\text {idle }}\right)}\right\}$

$$
\text { subject to } \begin{aligned}
\left(1+\frac{1}{3.5} \frac{\lambda_{\mathrm{MT}}}{\lambda_{\mathrm{BS}}}\right. & \left.\frac{1}{\left(\alpha_{\mathrm{M}}+\alpha_{\mathrm{S}}\left(1-\varepsilon\left(\lambda_{\mathrm{BS}}\right)\right)\right)}\right)^{-3.5} \\
& =\frac{p_{0}-\varepsilon\left(\lambda_{\mathrm{BS}}\right)}{p_{0}\left(1-\varepsilon\left(\lambda_{\mathrm{BS}}\right)\right)} \\
0 \leq \varepsilon\left(\lambda_{\mathrm{BS}}\right) \leq & p_{0} \leq 1
\end{aligned}
$$

where $\lambda_{\mathrm{BS}}^{\min }$ and $\lambda_{\mathrm{BS}}^{\max }$ are the minimum and maximum values that are allowed for $\lambda_{\mathrm{BS}}$, and we have made explicit that $\varepsilon$ depends on the density of the BSs, i.e., $\varepsilon=\varepsilon\left(\lambda_{\mathrm{BS}}\right)$.

The optimization problem $\mathcal{P}_{\lambda_{\mathrm{BS}}}$ is not conveniently formulated because of the difficulty of obtaining, from (40), an explicit expression of $\varepsilon$ as a function of $\lambda_{\mathrm{BS}}$. To overcome this issue, we formulate another optimization problem, $\mathcal{P}_{\varepsilon}$, as a function of $\varepsilon$ instead of $\lambda_{\mathrm{BS}}$, and prove, in the following proposition, that it is equivalent to the optimization problem $\mathcal{P}_{\lambda_{\mathrm{BS}}}$.

Proposition 6: Let $\varepsilon^{*}$ be the solution of the following optimization problem:

$$
\mathcal{P}_{\varepsilon}: \max _{\varepsilon \in\left[0, p_{0}\right]}\left\{\frac{\mathcal{F}(\varepsilon) \mathcal{G}(\varepsilon) \mathcal{Q}(\varepsilon)}{\left(\Delta P \mathcal{F}(\varepsilon)+P_{\text {idle }}\right)(1+c \mathcal{F}(\varepsilon))}\right\}
$$

where the following definitions hold:

$$
\begin{aligned}
& \mathcal{G} \triangleq \mathcal{G}(\varepsilon)=\alpha_{\mathrm{M}}+\alpha_{\mathrm{S}}(1-\varepsilon) \\
& \mathcal{F} \triangleq \mathcal{F}(\varepsilon)=1-\frac{p_{0}-\varepsilon}{p_{0}(1-\varepsilon)}=\frac{\varepsilon\left(1-p_{0}\right)}{p_{0}(1-\varepsilon)} \\
& \mathcal{H}(\varepsilon) \triangleq \frac{\lambda_{\mathrm{MT}}}{3.5}\left(\left(\frac{p_{0}-\varepsilon}{p_{0}(1-\varepsilon)}\right)^{-1 / 3.5}-1\right)^{-1} \\
& \mathcal{Q}(\varepsilon) \triangleq 1-\exp (-\pi w \mathcal{H}(\varepsilon)(1+c \mathcal{F}(\varepsilon)))
\end{aligned}
$$

Then, the solution of the optimization problem $\mathcal{P}_{\lambda_{\mathrm{BS}}}$ is the following:

$\lambda_{\mathrm{BS}}^{*} \triangleq \lambda_{\mathrm{BS}}\left(\varepsilon^{*}\right)$
$=\frac{\lambda_{\mathrm{MT}}}{3.5}\left(\left(\left(\frac{p_{0}-\varepsilon^{*}}{p_{0}\left(1-\varepsilon^{*}\right)}\right)^{-\frac{1}{3.5}}-1\right)\left(\alpha_{\mathrm{M}}+\alpha_{\mathrm{S}}\left(1-\varepsilon^{*}\right)\right)\right)^{-1}$

Proof: $\mathcal{P}_{\lambda_{\mathrm{BS}}}$ and $\mathcal{P}_{\varepsilon}$ admit a solution by virtue of Weierstrass extreme value theorem. $\mathcal{P}_{\varepsilon}$ is obtained directly from $\mathcal{P}_{\lambda_{\mathrm{BS}}}$ by formulating all the terms as a function of $\varepsilon$, and by noting that the left hand side of 40 is equal to $1-\mathcal{F}\left(\lambda_{\mathrm{BS}}\right)$. The equivalence of $\mathcal{P}_{\lambda_{\mathrm{BS}}}$ and $\mathcal{P}_{\varepsilon}$ follows because (40) is a bijection, i.e., the correspondence between $\lambda_{\mathrm{BS}}$ and $\varepsilon$ is oneto-one.

Based on Proposition 6, the following theorem yields the solution of $\mathcal{P}_{\lambda_{\mathrm{BS}}}$.

Theorem 2: Let $P_{\mathrm{tx}, \text { tot }}$ fulfill the following (sufficient) condition:

$$
P_{\mathrm{tx}, \mathrm{tot}} \geq \kappa \gamma_{\mathrm{A}} \sigma_{\mathrm{N}}^{2}\left(\frac{2(3.5)^{2}}{\pi \lambda_{\mathrm{MT}}} \frac{2\left(\exp \left(\lambda_{\mathrm{EP}}\right)-1\right)}{1+2\left(\exp \left(\lambda_{\mathrm{EP}}\right)-1\right)}\right)^{\beta / 2}
$$

The unique solution of $\mathcal{P}_{\lambda_{\mathrm{BS}}}$ is $\lambda_{\mathrm{BS}}^{*}$ in (47), where $0 \leq$ 
$\varepsilon^{*} \leq p_{0}$ is the unique solution of:

$$
\begin{aligned}
P_{\text {idle }} & =c \Delta P \mathcal{F}^{2}(\varepsilon)-\left(\Delta P \mathcal{F}(\varepsilon)+P_{\text {idle }}\right) \\
& \times(1+c \mathcal{F}(\varepsilon)) \frac{\mathcal{F}(\varepsilon)}{\dot{\mathcal{F}}(\varepsilon)}\left(\frac{\dot{\mathcal{G}}(\varepsilon)}{\mathcal{G}(\varepsilon)}+\frac{\dot{\mathcal{Q}}(\varepsilon)}{\mathcal{Q}(\varepsilon)}\right)
\end{aligned}
$$

Proof: See Appendix D

By direct inspection of Theorem 2, the following remarks can be made:

- The EE is a unimodal and strictly pseudo-concave function in $\varepsilon$ and $\lambda_{\mathrm{BS}}$ if the condition in (48) is fulfilled. The optimal deployment density of the BSs that maximizes the EE can be obtained by simply calculating the zero of a non-linear equation. We note, in particular, that $(48)$ is a sufficient condition for the strict pseudo-concavity of the EE.

- From [48, it is apparent that the EE is always strictly pseudo-concave if $\lambda_{\mathrm{EP}} \rightarrow 0$, i.e., (48) reduces to $P_{\text {tx,tot }} \geq 0$. This case study boils down to a single-tier cellular network where only the MBSs are available. Theorem 2, thus, is consistent with the analysis conducted in [1], where single-tier cellular networks and no renewable energy sources are considered.

- From (48), it is apparent that the EE is always strictly pseudo-concave if $\lambda_{\mathrm{MT}} \rightarrow \infty$, i.e., (48) reduces to $P_{\mathrm{tx}, \text { tot }} \geq 0$. This finding implies that, in the fully-loaded scenario, an optimal density of the BS exists for any choice of the system parameters.

- From (48), a similar trend is observed if $\gamma_{\mathrm{A}} \rightarrow 0$, i.e., the conventional definition of coverage probability is employed. It is worth noting that, in the presence of renewable energy sources, this case study is not trivial as that discussed in [1], since, in the present paper, $\varepsilon$ depends on $\lambda_{\mathrm{BS}}$, and the coverage probability in (19) is a function of $\lambda_{\mathrm{BS}}$.

- The solution stated in Theorem 2 can be simplified in some asymptotic operating regimes. A relevant case study is $\lambda_{\mathrm{MT}} / \lambda_{\mathrm{BS}} \ll 1$, which occurs in ultra-dense network deployments in which the density of the MTs is less than the density of the BSs. In this case, in fact, the left-hand side in, e.g., (37) reduces to $1-$ $\left(\lambda_{\mathrm{MT}} / \lambda_{\mathrm{BS}}\right)\left(\alpha_{\mathrm{M}}+\alpha_{\mathrm{S}}(1-\varepsilon)\right)^{-1}$, from which an explicit expression of $\varepsilon$ as a function of $\lambda_{\mathrm{BS}}$ is obtained. Case studies like this are not investigated in the present paper because of space limitations, but they provide evidence of the usefulness of the proposed analytical approach to get insight into the impact of renewable energy sources on the PSE and the EE of multi-tier cellular networks.

2) Half-Duplex Case Study: As anticipated, due to space limitations, we do not report the details of the half-duplex case study, but give only the final result in the following theorem.

Theorem 3: Consider the following optimization problem:

$$
\max _{\lambda_{\mathrm{BS}} \in\left[\lambda_{\mathrm{BS}}^{\min }, \lambda_{\mathrm{BS}}^{\max }\right]}\left\{\frac{\mathcal{F}\left(\lambda_{\mathrm{BS}}\right) \mathcal{G}\left(\lambda_{\mathrm{BS}}\right) \mathcal{Q}\left(\lambda_{\mathrm{BS}}\right) /\left(1+c \mathcal{F}\left(\lambda_{\mathrm{BS}}\right)\right)}{\left(\Delta P \mathcal{F}\left(\lambda_{\mathrm{BS}}\right)+P_{\text {idle }}\right)}\right\}
$$

$$
\begin{aligned}
& \text { subject to }\left(1+\frac{1}{3.5} \frac{\lambda_{\mathrm{MT}}}{\lambda_{\mathrm{BS}}} \frac{1}{\left(\alpha_{\mathrm{M}}+\alpha_{\mathrm{S}}\left(1-\varepsilon\left(\lambda_{\mathrm{BS}}\right)\right)\right)}\right)^{-3.5} \\
& =1-\frac{\left(1-p_{0}\right) \varepsilon\left(\lambda_{\mathrm{BS}}\right)}{1-\varepsilon\left(\lambda_{\mathrm{BS}}\right)} \\
& 0 \leq \varepsilon\left(\lambda_{\mathrm{BS}}\right) \leq\left(2-p_{0}\right)^{-1} \leq 1
\end{aligned}
$$

Let $P_{\mathrm{tx}, \text { tot }}$ fulfill the following (sufficient) condition:

$$
P_{\mathrm{tx}, \text { tot }} \geq \kappa \gamma_{\mathrm{A}} \sigma_{\mathrm{N}}^{2}\left(\frac{2(3.5)^{2}}{\pi \lambda_{\mathrm{MT}}} \frac{2 \exp \left(\lambda_{\mathrm{EP}}\right)-2}{3 \exp \left(\lambda_{\mathrm{EP}}\right)-2}\right)^{\beta / 2}
$$

The unique solution of the optimization problem in 51) is $\lambda_{\mathrm{BS}}^{*}$ given in the following equation:

$$
\begin{aligned}
& \lambda_{\mathrm{BS}}^{*} \triangleq \lambda_{\mathrm{BS}}\left(\varepsilon^{*}\right)=\frac{\lambda_{\mathrm{MT}}}{3.5} \\
& \times\left(\left(\left(1-\frac{\left(1-p_{0}\right) \varepsilon^{*}}{1-\varepsilon^{*}}\right)^{-\frac{1}{3.5}}-1\right)\left(\alpha_{\mathrm{M}}+\alpha_{\mathrm{S}}\left(1-\varepsilon^{*}\right)\right)\right)^{-1}
\end{aligned}
$$

where $0 \leq \varepsilon^{*} \leq\left(2-p_{0}\right)^{-1} \leq 1$ is the unique solution of the following non-linear equation:

$$
\begin{aligned}
P_{\text {idle }} & =c \Delta P \tilde{\mathcal{F}}^{2}(\varepsilon)-\left(\Delta P \tilde{\mathcal{F}}(\varepsilon)+P_{\text {idle }}\right)(1+c \tilde{\mathcal{F}}(\varepsilon)) \\
& \times \frac{\tilde{\mathcal{F}}(\varepsilon)}{\dot{\tilde{\mathcal{F}}}(\varepsilon)}\left(\frac{\dot{\mathcal{G}}(\varepsilon)}{\tilde{\mathcal{G}}(\varepsilon)}+\frac{\dot{\mathcal{Q}}(\varepsilon)}{\tilde{\mathcal{Q}}(\varepsilon)}\right)
\end{aligned}
$$

with $\tilde{\mathcal{G}}(\varepsilon) \triangleq \mathcal{G}(\varepsilon), \tilde{\mathcal{F}}(\varepsilon) \triangleq \mathcal{F}(\varepsilon)$, and:

$$
\begin{gathered}
\tilde{\mathcal{H}}(\varepsilon) \triangleq \frac{\lambda_{\mathrm{MT}}}{3.5}\left(\left(1-\frac{\varepsilon\left(1-p_{0}\right)}{1-\varepsilon}\right)^{-1 / 3.5}-1\right)^{-1} \\
\tilde{\mathcal{Q}}(\varepsilon) \triangleq 1-\exp (-\pi w \tilde{\mathcal{H}}(\varepsilon)(1+c \tilde{\mathcal{F}}(\varepsilon)))
\end{gathered}
$$

Proof: The proof is similar to Theorem 2 and is omitted due to space limitations.

We conclude this section by mentioning that the approach employed in Theorems 2 and 3 for optimizing the EE as a function of $\lambda_{\mathrm{BS}}$, i.e., formulating an equivalent optimization problem as a function of $\varepsilon$ that is easier to solve, can be used to analyze the impact of other system parameters, e.g., $p_{0}, \alpha_{\mathrm{M}}$, and $\alpha_{\mathrm{S}}$. Due to space limitations, these case studies are not analyzed in this paper, but the proposed approach seems to be general enough for unveiling the impact of other parameters. Simplified solutions may be obtained in some special cases (as discussed already), e.g., in fully-loaded $\left(\lambda_{\mathrm{MT}} / \lambda_{\mathrm{BS}} \gg 1\right)$ and lightly-loaded $\left(\lambda_{\mathrm{MT}} / \lambda_{\mathrm{BS}} \ll 1\right)$ cellular networks.

\section{Numerical and Simulation Results}

In this section, we show numerical results in order to substantiate the proposed approach and approximations for modeling multi-tier cellular networks empowered by renewable energy sources. The simulation setup is summarized in Table VI, and is in agreement with previous works on evaluating the EE in cellular networks [1]. It is worth noting that we consider a ratio between the association biases equal to 100 , in order to ensure that a sufficiently large number of MTs is associated to the SBSs, in order to compensate 
TABLE VI: Setup of parameters (unless otherwise stated)

\begin{tabular}{l|l}
\hline Parameter & Value \\
\hline$\beta$ & 3.5 \\
$\kappa=\left(4 \pi f_{c} / 3 \cdot 10^{8}\right)^{2}$ & $f_{c}=2.1 \mathrm{GHz}$ \\
$\mathrm{N}_{0}$ & $-174 \mathrm{dBm} / \mathrm{Hz}$ \\
$\mathrm{BW}_{\text {tot }}$ & $20 \mathrm{MHz}$ \\
$P_{\text {cir }}^{\mathrm{M}}$ & $51.14 \mathrm{dBm}$ \\
$P_{\text {idle }}$ & $48.75 \mathrm{dBm}$ \\
$P_{\text {tx }, \text { tot }}^{\mathrm{M}}$ & $43 \mathrm{dBm}$ \\
$P_{\text {tx }, \text { tot }}^{\mathrm{S}}$ & $23 \mathrm{dBm}$ \\
$\lambda_{\mathrm{BS}}=1 /\left(\pi \mathrm{R}_{\text {cell }}^{2}\right)$ & $\mathrm{R}_{\mathrm{cell}}=70 \mathrm{~m}$ \\
$\lambda_{\mathrm{MT}}=1 /\left(\pi \mathrm{R}_{\mathrm{MT}}^{2}\right)$ & $\mathrm{R}_{\mathrm{MT}}=51.29 \mathrm{~m}$ \\
$\gamma_{\mathrm{D}}=\gamma_{\mathrm{A}}$ & $5 \mathrm{~dB}$ \\
$\mathcal{L}, \lambda_{\mathrm{EP}}$ & $31,1.5($ packets per time-slot $)$ \\
$m^{\mathrm{L} 1}, m^{\mathrm{L} 2}$ & 3,2 \\
$m_{1}^{\mathrm{L} 1}, m_{1}^{\mathrm{L} 2}$ & 3,6 \\
$N_{\mathrm{R}}$ & 4 \\
$N_{\mathrm{Load}}^{\mathrm{MB}}, N_{\mathrm{Load}}^{\mathrm{S}}$ & 8,5 \\
$T_{\mathrm{M}}, T_{\mathrm{S}}$ & 100,1 \\
$\alpha_{\mathrm{M}}, \alpha_{\mathrm{S}}$ & 1,3 \\
\hline
\end{tabular}

for the smaller transmit power of the SBSs, and for the fact that the SBSs rely only on renewable energy sources for their operation. We study, however, the accuracy of our proposed approach for different values of the bias factors $\left(T_{\mathrm{M}}\right)$.

As far as Monte Carlo simulations are concerned, we implement the system model described in Section III without enforcing any of the modeling assumptions that are made for developing the analytical frameworks. Notably, the spatiotemporal correlations are not ignored. The simulator accounts for spatial and temporal dynamics as follows. First, the MBSs and SBSs are generated according to two independent PPPs. The system is then simulated for 1000 time slots, during which the spatio-temporal dynamics of the network are taken into account. Based on the assumption that time slot is chosen according to the mobility of the MTs (see Section II-B for details), independent realizations of the fast fading and the locations of the MTs are generated during each time slot. Also, in each time slot, the number of harvested power packets is generated according to a Poisson random variable. The performance metric of interest is computed at each time slot, and the average, over time and space, is calculated over the 1000 time slots. The procedure is iterated for $200-400$ spatial realizations (depending on the setup) for the locations of the MBSs and SBSs, and the (spatial) average is calculated.

In Figs. 1-6, we compare the PSE and EE that are obtained with our proposed approach against Monte Carlo simulations. We observe a good accuracy, and, in particular, analysis and simulations provide similar performance trends. Besides the good accuracy, we emphasize that the simulation results are difficult to generate and need longer time than our analytical approach. On average, the time that is needed to obtain the numerical simulations is at least 10,000 longer than the computation of the analytical formulas. In other words, it takes weeks to obtain the simulation results depicted in Figs. 16 , while it takes only a few minutes by using the analytical frameworks. The complexity and time of Monte Carlo methods
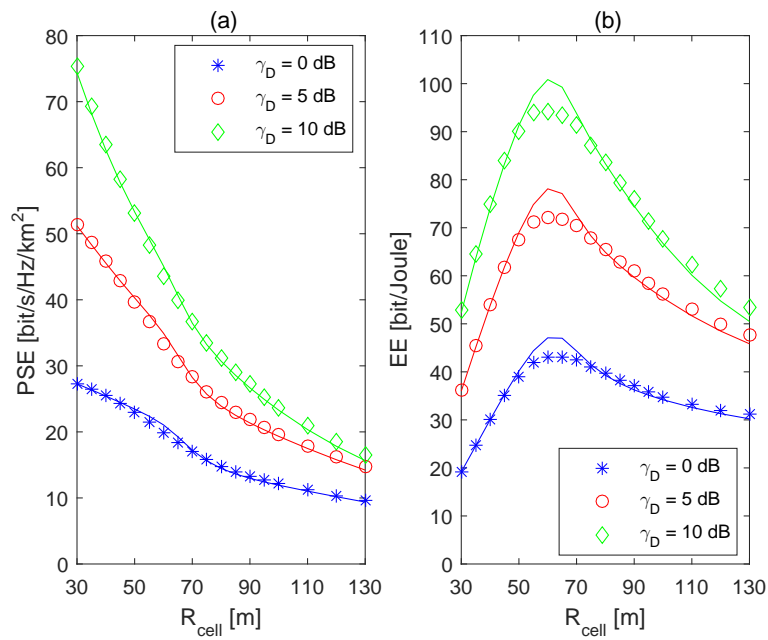

Fig. 1: PSE and EE vs. $R_{\text {cell }}$ and $\gamma_{\mathrm{D}}=\gamma_{\mathrm{A}}$. Setup: full-duplex, load model 1. Solid lines and markers show analytical frameworks and Monte Carlo simulations, respectively.

lie in the need of simulating the spatial dynamics of the network and the temporal dynamics that govern the energy harvesting. Even though our proposed approach is, in the most general case, semi-analytical due to the need of computing numerically the steady state probabilities, it advantages in terms of computation time and insights for system design, in contrast to Monte Carlo simulations, cannot be overlooked. Some gaps between analysis and Monte Carlo simulations exist, however, and they are mainly caused by the approximations employed to derive the analytical frameworks.

In particular: (i) from Figs. 1, 2, and 4, we evince that an optimal deployment density, transmit power, and association bias of the MBSs, respectively, is expected to exist, as proved in Section $\mathrm{V}$ in some case studies; (ii) in Figs. 4 and 6, we compare the optimal values of the transmit power and the association bias of the MBSs that are obtained from the analytical framework and Monte Carlo simulations. We observe that our approach well captures the optimal operating regime. The inaccuracy of Monte Carlo simulations is due to the long simulation time that is needed for estimating the optima; (iii) from Fig. 3, we evince that both the PSE and EE increase with $\lambda_{\mathrm{EP}}$. If $\lambda_{\mathrm{EP}}$ is large enough, the PSE and EE converge to a regime that is equivalent to a conventional two-tier cellular network without energy harvesting, i.e., all the SBSs are likely to have sufficient power to serve the MTs in their cells; and (iv) from Figs. 4 and 3, we note that the proposed approach is accurate for a large range of the bias factor and the average arrival rate of the power packets, respectively, which confirms that the temporal dynamics of the batteries of the SBSs are taken into account sufficiently well. Finally, even though not reported in the present paper due to space limitations, we have not noticed, for the considered setups, major differences (either quantitatively or qualitatively) between the half-duplex and full-duplex operating modes.

In Figs. 7 and 8, we leverage the analytical framework to investigate the role played by $\alpha_{\mathrm{M}}$ and $\alpha_{\mathrm{S}}$ as a function $\lambda_{\mathrm{BS}}$ and $\lambda_{\mathrm{EP}}$. We consider two case studies: (i) the constraint 

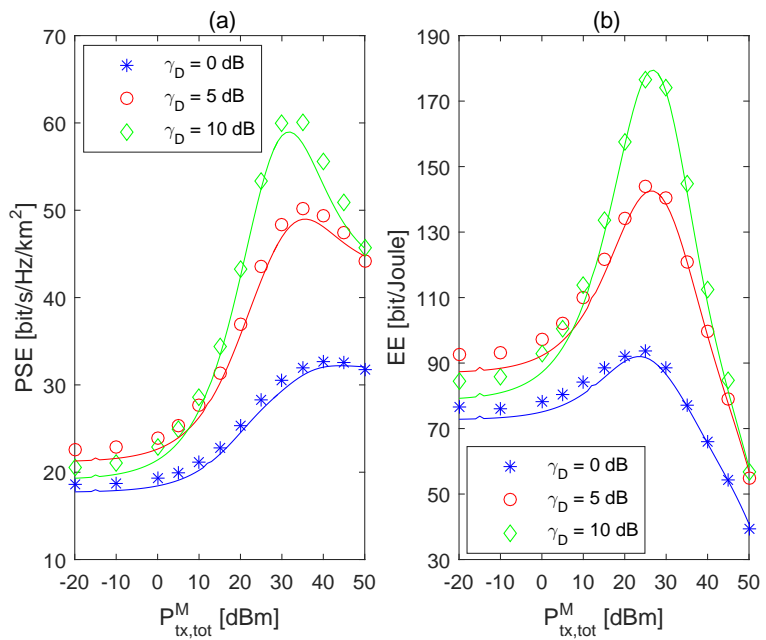

Fig. 2: PSE and EE vs. $P_{\mathrm{tx} \text {,tot }}^{\mathrm{M}}$ and $\gamma_{\mathrm{D}}=\gamma_{\mathrm{A}}$. Setup: full-duplex, load model 2. Solid lines and markers show analytical frameworks and Monte Carlo simulations, respectively.
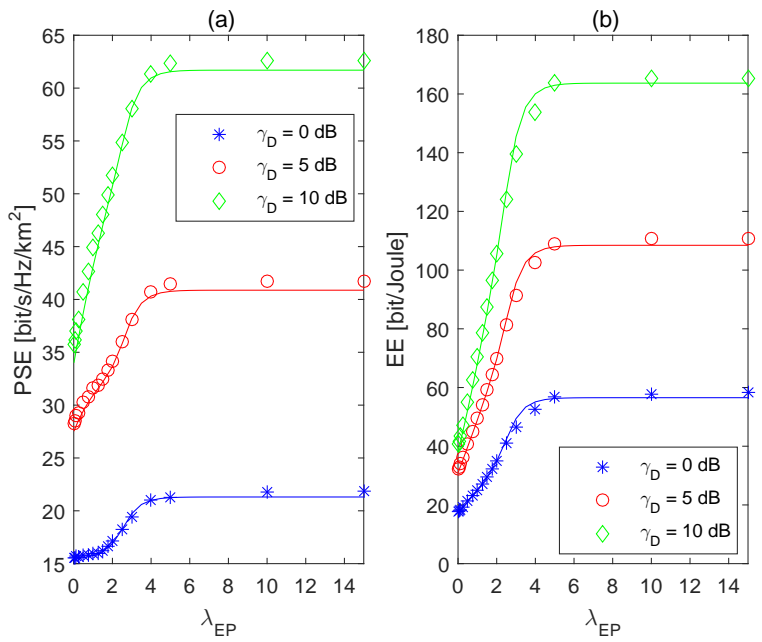

Fig. 3: PSE and EE vs. $\lambda_{\mathrm{EP}}$ and $\gamma_{\mathrm{D}}=\gamma_{\mathrm{A}}$. Setup: full-duplex, load model 2. Solid lines and markers show analytical frameworks and Monte Carlo simulations, respectively.

$\alpha_{\mathrm{M}}+\alpha_{\mathrm{S}}=1$ is enforced; and (ii) $\alpha_{\mathrm{M}}$ is kept fixed and $\alpha_{\mathrm{S}}$ takes different values. The first setup provides information on whether, by keeping $\lambda_{\mathrm{BS}}$ fixed, the density of the MBSs can be decreased while proportionally increasing the density of the SBSs, and the PSE and EE can still attain appropriate values even though the SBSs are powered only via renewable energy sources. The second setup provides information on whether dense deployments of SBSs enable us to improve the PSE and $\mathrm{EE}$ in cellular networks without the need of modifying the current deployments of MBSs.

In Fig. 7, we observe that the PSE decreases if $\alpha_{\mathrm{M}}$ decreases and $\alpha_{\mathrm{S}}$ increases but their sum is kept constant. Only if $\lambda_{\mathrm{EP}}$ is very large, the PSE tend to converge to that of a system model where all the BSs are MBSs, i.e., $\alpha_{\mathrm{S}}=0$. The increasing behavior of the PSE as a function of $\alpha_{\mathrm{S}}$ is due to the fact that the probability that the SBSs are available is approximately equal to one for large values of $\lambda_{\mathrm{EP}}$. If
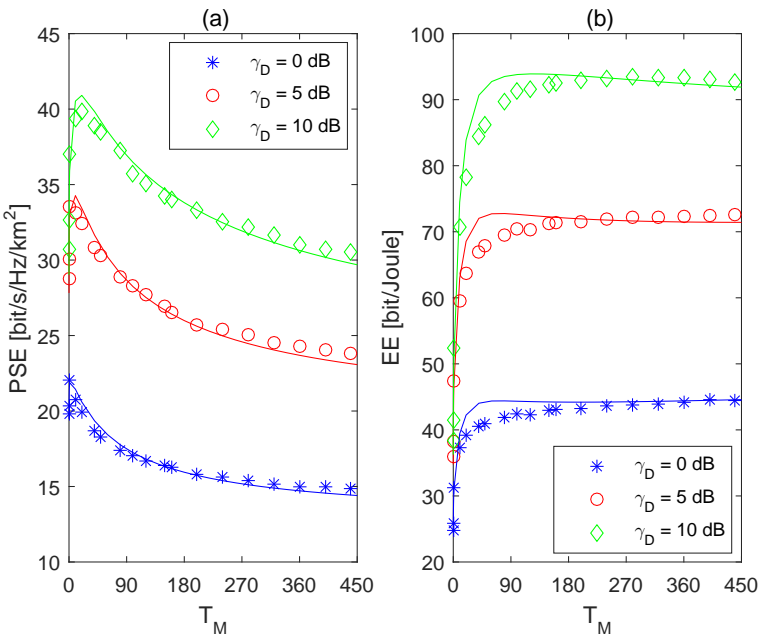

Fig. 4: PSE and EE vs. $T_{\mathrm{M}}$ and $\gamma_{\mathrm{D}}=\gamma_{\mathrm{A}}$. Setup: full-duplex, load model 1. Solid lines and markers show analytical frameworks and Monte Carlo simulations, respectively.
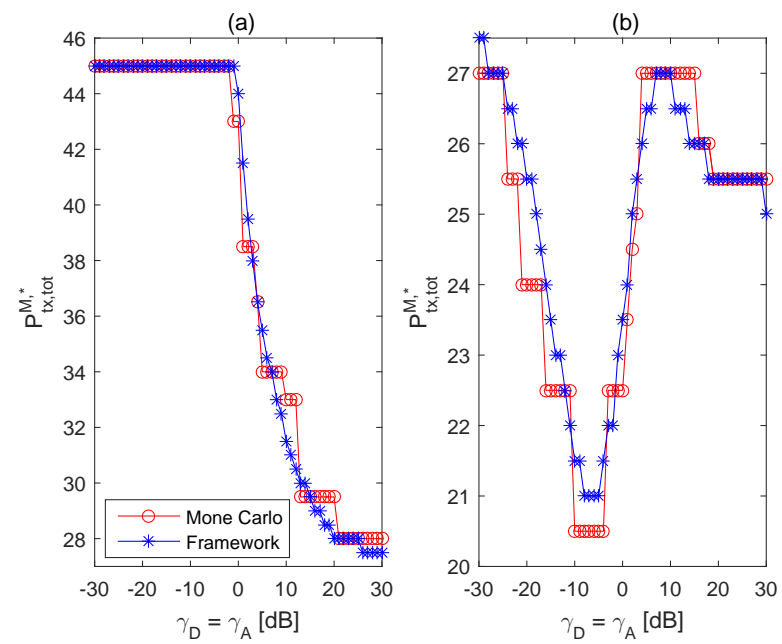

Fig. 5: Optimal $P_{\mathrm{tx}, \text { tot }}^{\mathrm{M}}$ that maximizes PSE (a) and EE (b). Setup: full-duplex, load model 2. Stars and circles show the analytical framework and Monte Carlo results, respectively.

$\lambda_{\mathrm{EP}}$ is not sufficiently large, on the other hand, the SBSs are likely not to be available and the PSE decreases. The $\mathrm{EE}$, on the other hand, always monotonically increases with $\alpha_{\mathrm{S}}$. By increasing $\alpha_{\mathrm{S}}$, in other words, we decrease the PSE but noticeably decrease the power consumption as well, since the latter is determined only by the MBSs. Therefore, the EE increases. This is another net example that reminds us that the EE is an efficiency metric given by the ratio of two functions. Optimizing the EE does not necessarily imply that the PSE is optimized as well. We conclude, therefore, that it is not sufficient to consider a single criterion of optimization, i.e., the EE. A multi-objective optimization problem, which jointly considers the PSE and the EE, is a more appropriate choice, and an interesting generalization of the results obtained in this paper and in [14] where no renewable energy sources are considered.

In Fig. 8 , we observe that by sufficiently increasing $\alpha_{S}$, 

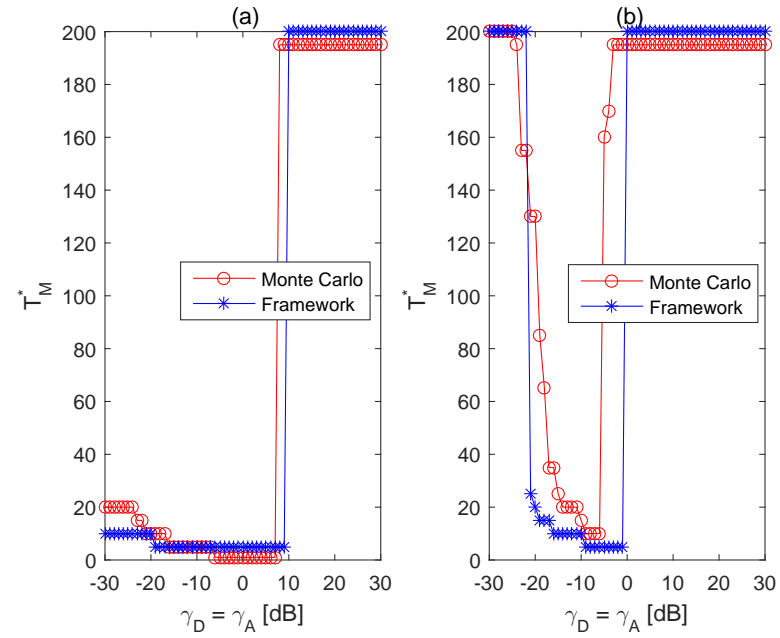

Fig. 6: Optimal $T_{M}$ that maximizes PSE (a) and EE (b). Setup: fullduplex, load model 2. Stars and circles show the analytical framework and Monte Carlo results, respectively.
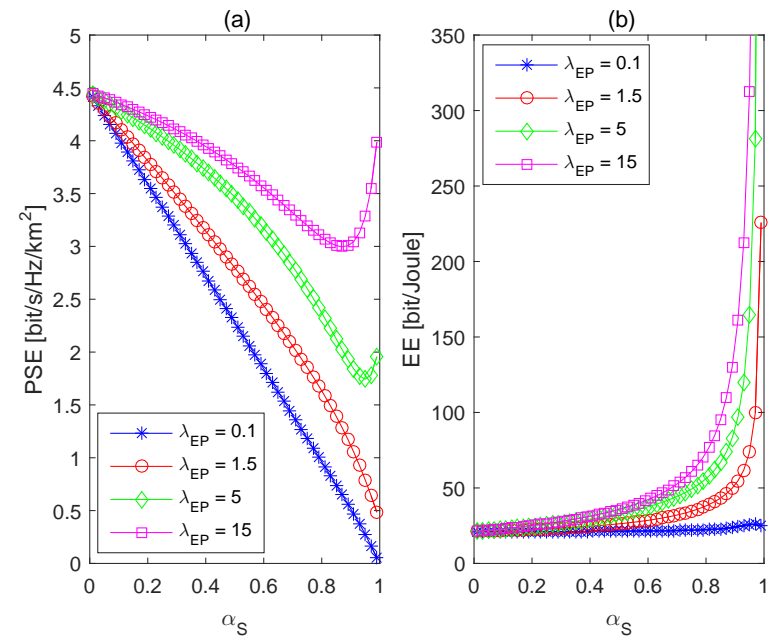

Fig. 7: PSE and EE vs. $\alpha_{\mathrm{S}}$ and $\gamma_{\mathrm{D}}=\gamma_{\mathrm{A}}=5 \mathrm{~dB}$. Scenario: $\alpha_{\mathrm{M}}+$ $\alpha_{\mathrm{S}}=1$ and $\mathrm{R}_{\text {cell }}=200 \mathrm{~m}$. The solid lines with markers show the analytical frameworks.

while keeping $\alpha_{\mathrm{M}}$ fixed, good performance can be obtained. In particular, both the PSE and EE increase as a function of $\alpha_{\mathrm{S}}$. Figure 8 highlights that the use of renewable energy sources is a promising solution in cellular networks as it enables them to obtain good PSE and EE at the same time. To achieve this target, however, a sufficiently large number of SBSs need to be deployed in order not to compromise the coverage probability of the typical MT. The coverage probability corresponding to Fig. 8 is depicted in Fig. 9. It can be noted that it first decreases and then increases, by reaching appropriate values if $\alpha_{\mathrm{S}}$ is sufficiently large. In contrast to the PSE and the EE that are network metrics, the coverage probability is related to the typical MT, and the insufficient deployment of SBSs may be deleterious for it. Figures 8 and 9 confirm that cellular networks empowered by renewable energy sources have great potential, but the trade-off between the performance of the typical user and the network need to be judiciously optimized.
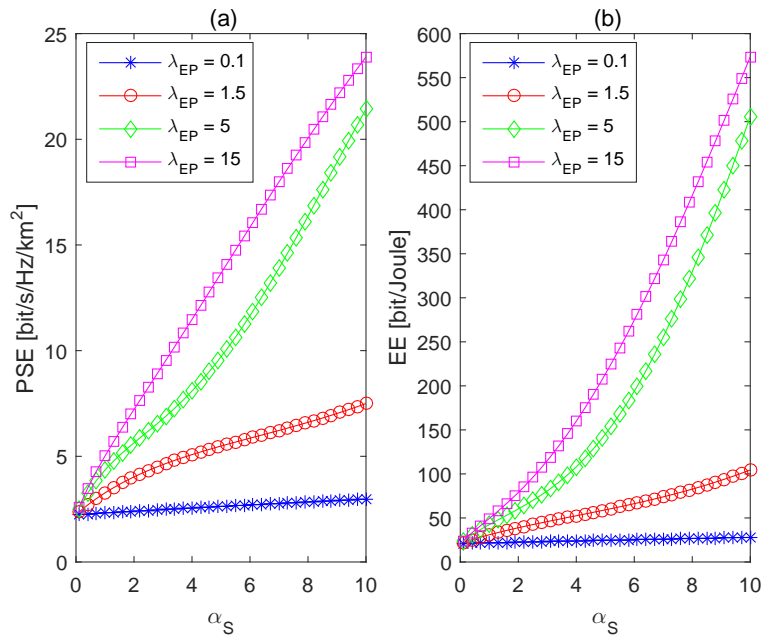

Fig. 8: PSE and EE vs. $\alpha_{\mathrm{S}}$ and $\gamma_{\mathrm{D}}=\gamma_{\mathrm{A}}=5 \mathrm{~dB}$. Scenario: $\alpha_{\mathrm{M}}=0.5$ and $R_{\text {cell }}=200 \mathrm{~m}$. The solid lines with markers show the analytical frameworks.

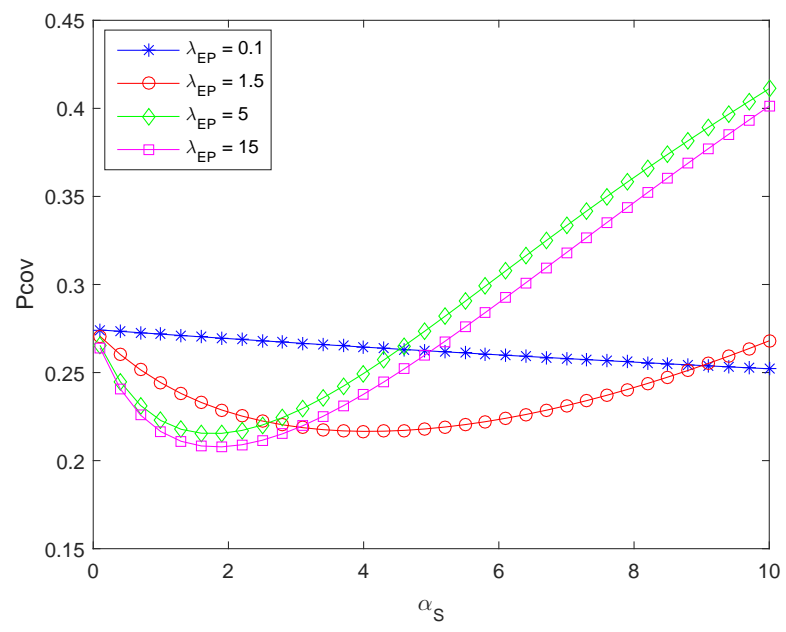

Fig. 9: Coverage probability vs. $\alpha_{\mathrm{S}}$ and $\gamma_{\mathrm{D}}=\gamma_{\mathrm{A}}=5 \mathrm{~dB}$. Scenario: $\alpha_{\mathrm{M}}=0.5$ and $\mathrm{R}_{\text {cell }}=200 \mathrm{~m}$. The solid lines with markers show the analytical frameworks.

In this case as well, therefore, embracing a multi-objective optimization framework seems to be a wise choice.

\section{CONCLUSION}

In this paper, we have proposed a new analytical approach for modeling, analyzing, and optimizing multi-tier cellular networks in which some base stations are powered solely through renewable energy sources. By capitalizing on the theory of Poisson point processes, tractable analytical expressions of the coverage probability, spectral efficiency, power consumption, and energy efficiency have been obtained. The proposed approach relies upon a few approximations that have been validated via Monte Carlo simulations. With the aid of the proposed analytical framework, some optimization problems have been formulated and comprehensively studied. It is proved, notably, that there exist optimal and unique values of the transmit power and density of the base stations that 
maximize the energy efficiency. This finding is rigorously proved under the assumption of a two-state model for the batteries of the base stations. Numerical results have confirmed these findings for more general system models. Finally, several important trends associated to the presence of renewable energy sources have been illustrated.

The proposed approach can be generalized in multiple ways. For example, multi-objective optimization problems can be formulated in order to capture the contrasting effects that renewable energy sources bring about in the design and optimization of cellular networks.

\section{APPENDIX A}

PROOF OF LEMMA 7

Let $\mathcal{P}$ denote a generic power level of the battery. Let us define $q \triangleq m_{1}^{\mathrm{L} 1}+N_{\mathrm{MT}} m^{\mathrm{L} 1}-1$ if $N_{\mathrm{MT}} \neq N_{\max }^{\mathrm{S}, d}$ and $q \triangleq \mathcal{L}-1$ if $N_{\mathrm{MT}}=N_{\max }^{\mathrm{S}, d}$, respectively. By definition, we have:

$$
\begin{aligned}
& P_{\text {serve }}^{\mathrm{S}}\left(d, o, N_{\mathrm{MT}}\right) \\
& \quad \triangleq \operatorname{Pr}\left\{m_{1}^{\mathrm{L} 1}+\left(N_{\mathrm{MT}}-1\right) m^{\mathrm{L} 1} \leq \mathcal{P} \leq q \mid \mathcal{P} \geq m_{1}^{\mathrm{L} 1}\right\} \\
& \stackrel{(a)}{=} \frac{\operatorname{Pr}\left\{m_{1}^{\mathrm{L} 1}+\left(N_{\mathrm{MT}}-1\right) m^{\mathrm{L} 1} \leq \mathcal{P} \leq q \cap \mathcal{P} \geq m_{1}^{\mathrm{L} 1}\right\}}{\operatorname{Pr}\left\{\mathcal{P} \geq m_{1}^{\mathrm{L} 1}\right\}} \\
& \quad=\frac{\operatorname{Pr}\left\{m_{1}^{\mathrm{L} 1}+\left(N_{\mathrm{MT}}-1\right) m^{\mathrm{L} 1} \leq \mathcal{P} \leq q\right\}}{\operatorname{Pr}\left\{\mathcal{P} \geq m_{1}^{\mathrm{L} 1}\right\}} \\
& \stackrel{(b)}{=} \frac{1}{1-\varepsilon_{d, o}}\left(\mathbf{1}\left(N_{\mathrm{MT}} \neq N_{\max }^{\mathrm{S}, d}\right) \sum_{s=s_{1}}^{s_{2}} v_{s}^{d, o}\right) \\
& +\frac{1}{1-\varepsilon_{d, o}}\left(\mathbf{1}\left(N_{\mathrm{MT}}=N_{\max }^{\mathrm{S}, d}\right) \sum_{s=s_{3}}^{s_{4}} v_{s}^{d, o}\right)
\end{aligned}
$$

where (a) follows from the fact that, by definition, $P_{\text {serve }}^{\mathrm{S}}\left(d, o, N_{\mathrm{MT}}\right)$ is conditioned upon the set of SBSs that are available, i.e., the normalization by $\operatorname{Pr}\left\{\mathcal{P} \geq m_{1}^{\mathrm{L} 1}\right\}$ is necessary; (b) follows by definition of steady state probabilities, from the identity $\operatorname{Pr}\left\{\mathcal{P} \geq m_{1}^{\mathrm{L} 1}\right\}=1-\varepsilon_{d, o}$, and defining $s_{1}=m_{1}^{\mathrm{L} 1}+\left(N_{\mathrm{MT}}-1\right) m^{\mathrm{L} 1}, s_{2}=m_{1}^{\mathrm{L} 1}+N_{\mathrm{MT}} m^{\mathrm{L} 1}-1$, $s_{3}=m_{1}^{\mathrm{L} 1}+\left(N_{\max }^{\mathrm{OFF}, d}-1\right) m^{\mathrm{L} 1}, s_{4}=\mathcal{L}-1$.

\section{APPENDIX B \\ PROOF OF PROPOSITION 1}

The proof of $P_{\mathrm{InA}}^{\mathrm{M}, \mathrm{L} 1}$ follows by noting that, by definition, $P_{\mathrm{InA}}^{\mathrm{M}, \mathrm{L} 1}=P_{\text {inactive }}^{\mathrm{L} 1}\left(\mathrm{M}, o, N_{\mathrm{RB}}\right)=\mathcal{I}_{\mathrm{RB}}\left(k, o, N_{\mathrm{RB}}\right)$ if $N_{\max }^{\mathrm{M}, d}=N_{\mathrm{RB}}$, and $P_{\mathrm{InA}}^{\mathrm{M}, \mathrm{L} 1}=P_{\text {inactive }}^{\mathrm{L} 1}\left(\mathrm{M}, o, N_{\text {Load }}^{\mathrm{M}}\right)=$ $\mathcal{I}_{\mathrm{MT}}\left(k, o, N_{\mathrm{Load}}^{\mathrm{M}}\right)$ if $N_{\max }^{\mathrm{M}, d}=N_{\mathrm{Load}}^{\mathrm{M}}$, respectively. The proof of $P_{\mathrm{InA}}^{\mathrm{S}, \mathrm{L} 1}$ follows by capitalizing on the total probability theorem as a function of the number of MTs that can be served due to the power level in the battery and the number of MTs associated with the SBS. More precisely, the following holds:

$$
P_{\mathrm{InA}}^{\mathrm{S}, \mathrm{L} 1}=\sum_{N_{\mathrm{MT}}=1}^{N_{\max }^{\mathrm{S}, \mathrm{L} 1}} P_{\text {inactive }}^{\mathrm{L} 1}\left(\mathrm{~S}, o, N_{\mathrm{MT}}\right) P_{\text {serve }}^{\mathrm{S}}\left(\mathrm{L} 1, o, N_{\mathrm{MT}}\right)
$$

which is obtained by considering every possible number of MTs that can be served as a function of the power level in the battery, and by taking into account the probability of occurrence of these events. The final result is obtained by using Lemmas 7, 9

\section{APPENDIX C \\ PROOF OF LEMMAS 10,13}

The proof of Lemma 10 follows from its definition, as follows:

$\operatorname{PSE}_{\mathrm{M}, \mathrm{L} 1}=\lambda_{\mathrm{MT}} \log _{2}\left(1+\gamma_{\mathrm{D}}\right) \mathrm{P}_{\mathrm{M}, \mathrm{L} 1}^{(\mathrm{cov})}\left(\gamma_{\mathrm{D}}, \gamma_{\mathrm{A}}\right)$
$\times\left(\sum_{e=0}^{N_{\mathrm{max}}^{\mathrm{M}, \mathrm{L} 1}-1} \frac{\mathrm{BW}_{\mathrm{tot}}}{N_{\mathrm{RB}}} \cdot 1 \cdot \bar{q}_{e}^{k}+\sum_{e=N_{\max }^{\mathrm{M}, \mathrm{L} 1}}^{+\infty} \frac{\mathrm{BW}}{N_{\mathrm{tot}}} \cdot \frac{N_{\mathrm{max}}^{\mathrm{M}, \mathrm{L} 1}}{e+1} \cdot \bar{q}_{e}^{k}\right)$

where the term in the brackets is obtained by applying the total probability theorem to the number of MTs that lie in a cell. In each summation: (i) the first term corresponds to the bandwidth allocated to an MT; (ii) the second term corresponds to the probability of scheduling an MT for transmission, which depends on the number of MTs in the cell and the available resources; and (iii) the third term corresponds to the probability of occurrence of a given number of MTs in the cell by conditioning on one MT being in the cell already. The proof follows from (8).

The proof of Lemma 11 follows from its definition, as:

$$
\begin{aligned}
& \mathrm{PSE}_{\mathrm{M}, \mathrm{L} 2}=\lambda_{\mathrm{MT}} \log _{2}\left(1+\gamma_{\mathrm{D}}\right) \mathrm{P}_{\mathrm{M}, \mathrm{L} 2}^{(\mathrm{cov})}\left(\gamma_{\mathrm{D}}, \gamma_{\mathrm{A}}\right) \\
& \times\left(\sum_{e=0}^{N_{\mathrm{Load}}^{\mathrm{M}}-1} \frac{\mathrm{BW}_{\mathrm{tot}}}{e+1} \cdot 1 \cdot \bar{q}_{e}^{k}+\sum_{e=N_{\mathrm{Load}}^{\mathrm{M}}}^{+\infty} \frac{\mathrm{BW}_{\mathrm{tot}}}{N_{\mathrm{Load}}^{\mathrm{M}}} \cdot \frac{N_{\mathrm{Load}}^{\mathrm{M}}}{e+1} \cdot \bar{q}_{e}^{k}\right) \\
& =\lambda_{\mathrm{MT}} \mathrm{BW}_{\mathrm{tot}} \log _{2}\left(1+\gamma_{\mathrm{D}}\right) \mathrm{P}_{\mathrm{M}, \mathrm{L} 2}^{(\mathrm{cov})}\left(\gamma_{\mathrm{D}}, \gamma_{\mathrm{A}}\right) \sum_{e=0}^{+\infty} \frac{1}{e+1} \cdot \bar{q}_{e}^{k}
\end{aligned}
$$

where the same rationale as for the proof of Lemma 10 is used. The difference is that the bandwidth allocated to the MTs (the first term in each summation) depends on the minimum between the number of MTs in the cell and the available resources. The proof follows from (8).

The proof of Lemma 12 follows from its definition, as:

$$
\begin{aligned}
& \mathrm{PSE}_{\mathrm{S}, \mathrm{L} 1}=\lambda_{\mathrm{MT}} \log _{2}\left(1+\gamma_{\mathrm{D}}\right) \mathrm{P}_{\mathrm{S}, \mathrm{L} 1}^{(\mathrm{cov})}\left(\gamma_{\mathrm{D}}, \gamma_{\mathrm{A}}\right) \\
& \times \sum_{N_{\mathrm{MT}}=1}^{N_{\text {max }}^{\mathrm{S}, \mathrm{L}}}\left(\sum_{e=0}^{N_{\mathrm{MT}}-1} \frac{\mathrm{BW}_{\mathrm{tot}}}{N_{\mathrm{RB}}} \cdot 1 \cdot \bar{q}_{e}^{k}+\sum_{e=N_{\mathrm{MT}}}^{+\infty} \frac{\mathrm{BW} \text { tot }}{N_{\mathrm{RB}}} \cdot \frac{N_{\mathrm{MT}}}{e+1} \cdot \bar{q}_{e}^{k}\right) \\
& \times P_{\text {serve }}^{\mathrm{S}}\left(\mathrm{L} 1, o, N_{\mathrm{MT}}\right)
\end{aligned}
$$

where the first summation accounts for the number of MTs that can be served as a function of the power levels of the batteries and the term in the brackets accounts for the number of MTs that lie in a cell. The two sums are obtained by applying the total probability theorem as a function of the latter two events. The rest of the proof is similar to the previous cases.

The proof of Lemma 13 follows from its definition, as:

$$
\begin{aligned}
& \mathrm{PSE}_{\mathrm{S}, \mathrm{L} 2}=\lambda_{\mathrm{MT}} \log _{2}\left(1+\gamma_{\mathrm{D}}\right) \mathrm{P}_{\mathrm{S}, \mathrm{L} 2}^{(\mathrm{cov})}\left(\gamma_{\mathrm{D}}, \gamma_{\mathrm{A}}\right) \\
& \times \sum_{N_{\mathrm{MT}}=1}^{N_{\text {max }}^{\mathrm{S} 2}}\left(\sum_{e=0}^{N_{\mathrm{MT}}-1} \frac{\mathrm{BW}_{\mathrm{tot}}}{e+1} \cdot 1 \cdot \bar{q}_{e}^{k}+\sum_{e=N_{\mathrm{MT}}}^{+\infty} \frac{\mathrm{BW} \text { tot }}{N_{\mathrm{MT}}} \cdot \frac{N_{\mathrm{MT}}}{e+1} \cdot \bar{q}_{e}^{k}\right) \\
& \times P_{\text {serve }}^{\mathrm{S}}\left(\mathrm{L} 2, o, N_{\mathrm{MT}}\right)
\end{aligned}
$$




$$
\begin{aligned}
& =\lambda_{\mathrm{MT}} \log _{2}\left(1+\gamma_{\mathrm{D}}\right) \mathrm{BW}_{\mathrm{tot}}\left(\sum_{e=0}^{+\infty} \frac{1}{e+1} \cdot \bar{q}_{e}^{k}\right) \\
& \times\left(\sum_{N_{\mathrm{MT}}=1}^{N_{\text {max }}^{\mathrm{S} \text { L2 }}} P_{\text {serve }}^{\mathrm{S}}\left(\mathrm{L} 2, o, N_{\mathrm{MT}}\right)\right) \mathrm{P}_{\mathrm{S}, \mathrm{L} 2}^{(\text {cov })}\left(\gamma_{\mathrm{D}}, \gamma_{\mathrm{A}}\right)
\end{aligned}
$$

where the same rationale as in the previous case is employed. The proof follows by using the identity $\sum_{N_{\mathrm{MT}}=1}^{N_{\max }^{\mathrm{S}} 2} P_{\text {serve }}^{\mathrm{S}}\left(\mathrm{L} 2, o, N_{\mathrm{MT}}\right)=1$ along with 8 .

\section{APPENDIX D \\ PROOF OF THEOREM 2}

Since the proof is lengthly, we report only its main idea and omit the algebraic details. The proof follows by taking into account that: (i) $\mathcal{G}(\varepsilon)$ is an affine and monotonically decreasing function in $\varepsilon$; (ii) $\mathcal{F}(\varepsilon)$ is a monotonically increasing and convex function in $\varepsilon$; and (iii) $\mathcal{Q}(\varepsilon)$ is a monotonically decreasing function in $\varepsilon$. Also, it is concave in $\varepsilon$ if the condition $\mathcal{C}_{1}: P_{\text {tx }, \text { tot }} \geq \kappa \gamma_{\mathrm{A}} \sigma_{\mathrm{N}}^{2}\left(2(3.5)^{2} /\left(\pi \lambda_{\mathrm{MT}}(1+c)\right)\right)^{\beta / 2}$ is fulfilled. The findings (i) and (ii) originate by direct inspection of the functions. The finding in (iii) originates by computing the first-order and second-order derivatives of $\mathcal{Q}(\varepsilon)$ as a function of $\varepsilon$. If $0 \leq \varepsilon^{*} \leq p_{0}$, the first-order derivative is negative. It is not easy to prove that the second-order derivative is negative for every parameters. A sufficient condition that makes the latter derivative negative for $0 \leq \varepsilon^{*} \leq p_{0}$ is when the system parameters fulfill $\mathcal{C}_{1}$. The proof follows by computing the firstorder derivative of the $\mathrm{EE}$ as a function of $\varepsilon$, and by proving that sufficient conditions for it to have a unique zero, which is the maximizer of the EE, are that $\mathcal{C}_{1}$ and $\mathcal{C}_{2}:(1 /(1+c))^{\beta / 2} \leq$ $\left(2\left(\exp \left(\lambda_{\mathrm{EP}}\right)-1\right) /\left(1+2\left(\exp \left(\lambda_{\mathrm{EP}}\right)-1\right)\right)\right)^{\beta / 2}$ are fulfilled simultaneously. By combining $\mathcal{C}_{1}$ and $\mathcal{C}_{2}$, we obtain (48). Finally, (49) is obtained by equating the first-order derivative of the EE to zero.

\section{REFERENCES}

[1] M. Di Renzo, A. Zappone, T. T. Lam, and M. Debbah, "System-level modeling and optimization of the energy efficiency in cellular networks - A stochastic geometry framework", IEEE Trans. Wireless Commun., vol. 17, no. 4, pp. 2539-2556, Apr. 2018.

[2] J. G. Andrews, F. Baccelli, and R. K. Ganti, "Seven ways that HetNets are a cellular paradigm shift", IEEE Commun. Mag., vol. 51, no. 3, pp. 136-144, Mar. 2013.

[3] G. Piro, M. Miozzo, F. Forte, N. Baldo, L. A. Grieco, and P. Dini, "HetNets powered by renewable energy sources: Sustainable nextgeneration cellular networks", IEEE Internet Comput., vol. 17, no. 1, pp. 32-39, Jan./Feb. 2013

[4] J. G. Andrews, F. Baccelli, and R. K. Ganti, "A tractable approach to coverage and rate in cellular networks", IEEE Trans. Commun., vol. 59, no. 11, pp. 3122-3134, Nov. 2011.

[5] H. S. Dhillon, Y. Li, P. Nuggehalli, Z. Pi, and J. G. Andrews, "Fundamentals of heterogeneous cellular networks with energy harvesting", IEEE Trans. Wireless Commun., vol. 13, no. 5, pp. 2782-2797, May. 2014.

[6] K. Huang, M. Kountouris, and V. O. K. Li, "Renewable powered cellular networks: Energy field modeling and network coverage", IEEE Trans. Wireless Commun., vol. 14, no. 8, pp. 4234-4247, Aug. 2015.

[7] A. H. Sakr and E. Hossain, "Analysis of K-tier uplink cellular networks with ambient RF energy harvesting", IEEE J. Sel. Areas Commun. vol. 33, no. 10, pp. 2226-2238, Oct. 2015.

[8] P.-S. Yu, J. Lee, T. Quek, Y.-W. P. Hong, "Traffic offloading in heterogeneous networks with energy harvesting personal cells - Network throughput and energy efficiency", IEEE Trans. Wireless Commun., vol. 15, no. 2, pp. 1146-1161, Feb. 2016.
[9] F. Parzysz, M. Di Renzo, and C. Verikoukis, "Power-availability-aware cell association for energy-harvesting small-cell base stations", IEEE Trans. Wireless Commun., vol. 16, no. 4, pp. 2409-2422, Apr. 2017.

[10] H. Wu, X. Tao, N. Zhang, D. Wang, S. Zhang, and X. Shen, "On base station coordination in cache- and energy harvesting-enabled HetNets: A stochastic geometry study", IEEE Trans. Commun., vol. 66, no. 7, pp. 3079-3091, Jul. 2018.

[11] M. A. Kishk and H. S. Dhillon, "Joint uplink and downlink coverage analysis of cellular-based RF-powered IoT network", IEEE Trans. Green Commun. Netw., vol. 2, no. 2, pp. 446-459, Jun. 2018.

[12] K. Liu and T. Yu, "Performance of off-grid small cells with non-uniform deployment in two-tier HetNet", IEEE Trans. Wireless Commun., vol. 17, no. 9, pp. 6135-6148, Sep. 2018.

[13] M. Di Renzo, T. T. Lam, A. Zappone, and M. Debbah, "A tractable closed-form expression of the coverage probability in Poisson cellular networks", IEEE Wireless Commun. Lett., vol. 1, no. 1, pp. 249-252, Feb. 2019.

[14] M. Di Renzo, A. Zappone, T. T. Lam, and M. Debbah, "Spectral-energy efficiency Pareto front in cellular networks: A stochastic geometry framework", IEEE Wireless Commun. Lett., vol. 8, no. 2, pp. 424-427, Apr. 2019.

[15] P. Lee, Z. A. Eu, M. Han, and H. Tan, "Empirical modeling of a solar-powered energy harvesting wireless sensor node for time-slotted operation", IEEE Wireless Commun. Netw. Conf., May 2011.

[16] G. Chisci, H. ElSawy, A. Conti, M.-S. Alouini, and M. Z. Win, "Uncoordinated massive wireless networks: Spatiotemporal models and multiaccess strategies", IEEE/ACM Trans. Netw., vol. 27, no. 3, pp. 918931, Jun. 2019

[17] G. Chisci , H. ElSawy, A. Conti, M.-S. Alouini, and M. Z. Win, "Latency in downlink cellular networks with random scheduling", IEEE Int. Conf. Commun., May 2019.

[18] Y. Wang, H. H. Yang, Q. Zhu, and T. Q. S. Quek, "Analysis of packet throughput in spatiotemporal HetNets with scheduling and various traffic loads", IEEE Wireless Commun. Lett., vol. 9, no. 1, pp. 95-98, Jan. 2020.

[19] M. Gharbieh, H. ElSawy, A. Bader, and M. S. Alouini, "Spatiotemporal stochastic modeling of IoT enabled cellular networks: Scalability and stability analysis", IEEE Trans. Commun., vol. 65, no. 8, pp. 3585-3600, Aug. 2017.

[20] M. Gharbieh, H. ElSawy, H. C. Yang, A. Bader, and M. S. Alouini, "Spatiotemporal model for uplink IoT traffic: Scheduling and random access paradox", IEEE Trans. Wireless Commun., vol. 17, no. 12, pp. 8357-8372, Dec. 2018

[21] H. H. Yang and T. Q. S. Quek, Spatio-temporal analysis for SINR coverage in small cell networks", IEEE Trans. Commun., vol. 67, no. 8, pp. 5520-5531, Aug. 2019.

[22] M. Di Renzo and P. Guan, "Stochastic geometry modeling and systemlevel analysis of uplink heterogeneous cellular networks with multiantenna base stations", IEEE Trans. Commun., vol. 64, no. 6, pp. 24532476, Jun. 2016.

[23] M. Di Renzo, A. Guidotti, and G. E. Corazza, "Average rate of downlink heterogeneous cellular networks over generalized fading channels - A stochastic geometry approach", IEEE Trans. Commun., vol. 61, no. 7, pp. 3050-3071, Jul. 2013.

[24] S. M. Yu and S. L. Kim, "Downlink capacity and base station density in cellular networks", IEEE Work. Spatial Stochastic Models for Wireless Networks, Tsukuba Science City, Japan, pp. 1-7, May 2013.

[25] S. Singh, H. S. Dhillon, and J. G. Andrews, "Offloading in heterogeneous networks: Modeling, analysis, and design insights", IEEE Trans. Wireless Commun., vol. 12, no. 5, pp. 2484-2497, May 2013.

[26] J. G. Kemeny and J. L. Snell, Finite Markov Chains, Dec. 1960. 


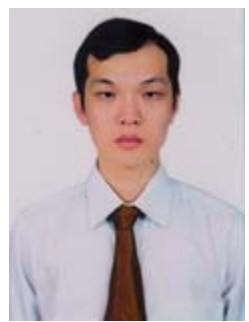

Thanh Tu Lam (S'15) was born in Ho Chi Minh, Vietnam, in 1986. He received the B.Sc. degree in electronics and telecommunications engineering from Ho Chi Minh University of Technology, Vietnam, in 2009, and the M.Sc. degree in telecommunications engineering from the Posts and Telecommunications Institute of Technology, Vietnam, in 2014. During the period 2015-2018, he was with French National Center for Scientific Research (CNRS), Paris, France, as an Early Stage Researcher of the European-funded project $\mathrm{H} 2020$ ETN-5Gwireless. In 2018, he received the Ph.D. degree from the Laboratory of Signals and Systems of Paris-Saclay University, Paris, France. Currently, he is a Research Fellow in the Xlim Laboratory of Poitiers University, Limoges, France. His research interests include wireless communications and stochastic geometry.

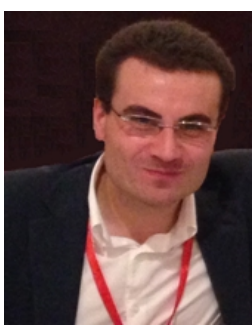

Marco Di Renzo (S'05-AM'07-M'09-SM'14-F'20) was born in L'Aquila, Italy, in 1978. He received the Laurea (cum laude) and Ph.D. degrees in electrical engineering from the University of L'Aquila, Italy, in 2003 and 2007, respectively, and the Habilitation a Diriger des Recherches (Doctor of Science) degree from University Paris-Sud, France, in 2013.

Since 2010, he has been with the French National Center for Scientific Research (CNRS), where he is a CNRS Research Director (CNRS Professor) in the Laboratory of Signals and Systems (L2S) of ParisSaclay University CNRS and CentraleSupelec, Paris, France. He is a Nokia Foundation Visiting Professor at Aalto University, Helsinki, Finland, and a Honorary Professor at University Technology Sydney, Sydney, Australia.

$\mathrm{He}$ serves as the Editor-in-Chief of IEEE Communications Letters. He served as an Editor of IEEE Transactions on Communications, IEEE Transactions on Wireless Communications, IEEE Communications Letters, and as the Associate Editor-in-Chief of IEEE Communications Letters. He is a Distinguished Lecturer of the IEEE Vehicular Technology Society and IEEE Communications Society.

$\mathrm{He}$ is a recipient of several awards, including the 2013 IEEE-COMSOC Best Young Researcher Award for Europe, Middle East and Africa, the 2013 NoE-NEWCOM\# Best Paper Award, the 2014-2015 Royal Academy of Engineering Distinguished Visiting Fellowship, the 2015 IEEE Jack Neubauer Memorial Best System Paper Award, the 2015 CNRS Award for Excellence in Research and Ph.D. Supervision, the 2016 MSCA Global Fellowship (declined), the 2017 SEE-IEEE Alain Glavieux Award, the 2018 IEEECOMSOC Young Professional in Academia Award, and 8 Best Paper Awards at IEEE conferences (2012 and 2014 IEEE CAMAD, 2013 IEEE VTC-Fall, 2014 IEEE ATC, 2015 IEEE ComManTel, 2017 IEEE SigTelCom, EAI 2018 INISCOM, IEEE ICC 2019).

$\mathrm{He}$ is a Highly Cited Researcher (Clarivate Analytics, Web of Science) and a Fellow of the IEEE. 